\title{
A comparison of fourteen fully characterized mammalian- associated Campylobacter fetus isolates suggests that loss of defense mechanisms contribute to high genomic plasticity and subspecies evolution
}

Susan A Nadin-Davis ${ }^{\text {Corresp., } 1}$, John Chmara ${ }^{1}$, Catherine D Carrillo ${ }^{2}$, Kingsley Amoako ${ }^{3}$, Noriko Goji ${ }^{3}$, Marc-Olivier Duceppe $^{1}$, John Devenish ${ }^{1}$

1 Ottawa Laboratory Fallowfield, Canadian Food Inspection Agency, Ottawa, Ontario, Canada

2 Ottawa Laboratory Carling, Canadian Food Inspection Agency, Ottawa, Ontario, Canada

3 National Centre for Animal Diseases, Canadian Food Inspection Agency, Lethbridge, Alberta, Canada

Corresponding Author: Susan A Nadin-Davis

Email address: nadindavis@gmail.com

Campylobacter fetus is currently classified into three main subspecies, but only two of these, $C$. fetus subspecies fetus and $C$. fetus subsp. venerealis originate principally from ruminants where they inhabit different niches and cause distinct pathogenicity. Their importance as pathogens in international trade and reporting is also different yet the criteria defining these properties have never been fully substantiated nor understood. The situation is further compromised because the ability to differentiate between these two closely related $C$. fetus subspecies has traditionally been performed by phenotypic characterisation of isolates, methods which are limited in scope, time-consuming, tedious, and often yield inconsistent results, thereby leading to isolate misidentification. The development of robust genetic markers that could enable rapid discrimination between $C$. fetus subsp. fetus and subsp. venerealis has also been challenging due to limited differences in the gene complement of their genomes, high levels of sequence repetition, the small number of closed genome sequences available and the lack of standardisation of the discriminatory biochemical tests employed for comparative purposes. To yield a better understanding of the genomic differences that define these $C$. fetus strains, seven isolates were exhaustively characterised phenotypically and genetically and compared with seven previously well characterised isolates. Analysis of these $14 \mathrm{C}$. fetus samples clearly illustrated that adaption by $C$. fetus subsp. venerealis to the bovine reproductive tract correlated with increasing genome length and plasticity due to the acquisition and propagation of several mobile elements including prophages, transposons and plasmids harbouring virulence factors. Significant differences in the repertoire of the CRISPR (clustered regularly interspersed short palindromic repeats)-cas system of all $C$. fetus 
strains was also found. We therefore suggest that a deficiency in this adaptive immune system may have permitted the emergence of extensive genome plasticity and led to changes in host tropism through gene disruption and / or changes in gene expression. Notable differences in the sub-species complement of DNA adenine methylase genes may also have an impact. These data will facilitate future studies to better understand the precise genetic differences that underlie the phenotypic and virulence differences between these animal pathogens and may identify additional markers useful for diagnosis and subtyping. 
1 A comparison of fourteen fully characterized mammalian-associated

2

\section{(7)}

\section{Campylobacter fetus isolates suggests that loss of defense}

\section{mechanisms contribute to high genomic plasticity and subspecies}

\section{evolution}

Susan A. Nadin-Davis ${ }^{1}$, John Chmara ${ }^{1}$, Catherine Carrillo ${ }^{2}$, Kingsley Amoako ${ }^{3}$, Noriko Goji ${ }^{3}$,

Marc-Olivier Duceppe ${ }^{1} \&$ John Devenish ${ }^{1}$

${ }^{1}$ Ottawa Laboratory Fallowfield, Canadian Food Inspection Agency, Ottawa, Ontario, Canada; ${ }^{2}$ Ottawa Laboratory Carling, Canadian Food Inspection Agency, Ottawa, Ontario, Canada;

${ }^{3}$ National Centre for Animal Diseases, Canadian Food Inspection Agency, Lethbridge, Alberta, Canada

Corresponding Author:

Susan Nadin-Davis

Email address: nadindavis@gmail.com

Short title: C. fetus genomic variation 


\section{Abstract}

24 Campylobacter fetus is currently classified into three main subspecies, but only two of these, $C$.

25 fetus subspecies fetus and C. fetus subsp. venerealis originate principally from ruminants where

26 they inhabit different niches and cause distinct pathogenicity. Their importance as pathogens in

27 international trade and reporting is also different yet the criteria defining these properties have

28 never been fully substantiated nor understood. The situation is further compromised because the ability to differentiate between these two closely related $C$. fetus subspecies has traditionally been performed by phenotypic characterisation of isolates, methods which are limited in scope, time-consuming, tedious, and often yield inconsistent results, thereby leading to isolate misidentification. The development of robust genetic markers that could enable rapid discrimination between $C$. fetus subsp. fetus and subsp. venerealis has also been challenging due to limited differences in the gene complement of their genomes, high levels of sequence repetition, the small number of closed genome sequences available and the lack of standardisation of the discriminatory biochemical tests employed for comparative purposes. To yield a better understanding of the genomic differences that define these $C$. fetus strains, seven isolates were exhaustively characterised phenotypically and genetically and compared with seven previously well characterised isolates. Analysis of these 14 C. fetus samples clearly illustrated that adaption by $C$. fetus subsp. venerealis to the bovine reproductive tract correlated with increasing genome length and plasticity due to the acquisition and propagation of several mobile elements including prophages, transposons and plasmids harbouring virulence factors. Significant differences in the repertoire of the CRISPR (clustered regularly interspersed short palindromic repeats)-cas system of all $C$. fetus strains was also found. We therefore suggest that a deficiency in this adaptive immune system may have permitted the emergence of extensive genome plasticity and led to changes in host tropism through gene disruption and / or changes in gene expression. Notable differences in the sub-species complement of DNA adenine methylase genes may also have an impact. These data will facilitate future studies to better understand the precise genetic differences that underlie the phenotypic and virulence differences between these animal pathogens and may identify additional markers useful for diagnosis and sub-typing. 
51

52

53

54

55

56

57

58

59

60

61

62

63

64

65

66

67

68

69

70

71

72

73

74

75

76

77

78

79

80

81

82

83

84

85

86

87

88

89

90

\section{Introduction}

The Campylobacter genus comprises a diverse and emerging group of Gram negative, curved and spiral rod shaped bacteria (Fitzgerald 2015) currently divided into 44 distinct species by the List of Prokaryotic names with Standing in Nomenclature (LPSN-accessed April 17, 2020) (Parte 2018) while potential new members continue to be described (Silva et al. 2020). In this genus, three subspecies of C. fetus are recognised, one of which, the genetically divergent $C$. fetus subsp. testudinum (Gilbert et al. 2016), is normally associated with reptilian hosts only (Fitzgerald et al. 2014) and will not be discussed further here (Sprenger et al. 2012). The other 2 subspecies, C. fetus subsp. fetus (CFF) and C. fetus subsp. venerealis (CFV), can inhabit both the gastrointestinal or reproductive tracts of ruminants including cattle (Sprenger et al. 2012). Strains of CFF have been reported as the cause of serious septicaemic infections in humans. This is observed especially in elderly patients or those with an underlying medical condition involving some form of immunodeficiency (Gazaigne et al. 2008; Pacanowski et al. 2008). CFV has been isolated from humans on rare occasions but its role in disease is only suspected because identification to the subspecies level is not conducted in many human infections caused by $C$. fetus (Wagenaar et al. 2014). The usual mode of transmission to humans is through ingestion or by injection of contaminated medical products. As such, CFF and CFV are considered to be accidental and opportunistic pathogens of humans that act as dead end hosts. It is believed that the fastidious nature of this microorganism results in significant underreporting of its prevalence and its role in disease (van Bergen et al. 2008) and the serious nature of CFF, and possibly CFV, infections in humans makes them a public health concern (Butzler 2004; Woo et al. 2002).

Regarding pathogenesis in ruminants, disease and host affiliation, CFF is found mainly in the intestinal tract of cattle, sheep and other ruminants and is believed to spread through the fecal/oral route by grazing on infected fields where the resulting ingestion back into the intestinal tract causes a descending systemic infection which localizes in the placental tissues in pregnant ewes and cows resulting in storm abortions in sheep and occasional abortion in cattle (Sprenger et al. 2012). CFV is found only in the reproductive tract of cattle but its classification is further complicated by the description of a distinct biovar, $C$. fetus subsp. venerealis biovar intermedius (CFVi) which, unlike CFV, is not confined solely to the genital tract of cattle but has also been isolated from the intestinal tract (Florent 1963). CFVi is aligned with CFV solely because of the same subspecies designation. In contrast to CFF, both CFV, and by association CFVi, are considered venereally transmitted through natural mating or by contaminated semen or equipment and, in contrast to $\mathrm{CFF}$, produce an ascending, non systemic, infection in cows with the main manifestation of disease being infertility and, occasionally, early embryonic death leading to abortion in pregnancy. It is the condition of infertility and venereal spread caused by CFV and CFVi, not oral systemic spread and abortion caused by CFF, which defines the disease bovine genital campylobacteriosis (BGC). BGC is highly prevalent in some countries, including several in South America (Silveira et al. 2018), and is associated with high economic losses (Sprenger et al. 2012; Veron \& Chatelain 1973). In other countries, including Canada, reporting 
91

of this disease is rare but CFV and CFVi are occasionally isolated by culture screening of bulls serviced at AI facilities. As a result of the serious nature of $\mathrm{BGC}$ and its world-wide distribution, the World Organization for Animal Health (OIE) has designated BGC, and by association CFV/CFVi, a listed notifiable disease important in international trade (OIE 2018) and this has not changed for many years.

However, gaps and contradictions in the scientific literature and knowledge regarding infertility and BGC exist and have remained for as long as BGC has been described. The role of CFVi in ruminant disease has never been truly elucidated even in official reference texts (Wagenaar \& van der Graaf-van Bloois 2018). In addition, attempts to verify infertility using manual introduction of CFV into the vulva of cows failed with no attempt to test CFF strains in parallel (Clark 1971). Other studies showed that both type $\mathrm{A}\left(\mathrm{H}_{2} \mathrm{~S}\right.$ and $1 \%$ Glycine growth tolerance negative resembling CFV) and type $\mathrm{B}$ strains $\left(\mathrm{H}_{2} \mathrm{~S}\right.$ positive and $1 \%$ Glycine growth tolerant resembling $\mathrm{CFF}$ ) could be isolated from heifers with infertility that were originally inseminated with semen from bulls known to have low fertility problems (Park et al. 1962). Furthermore, it was reported that serovar B strains of CFF could cause bovine infertility in about $5 \%$ of observed infections caused by C. fetus ssp. (Dekeyser 1984). This type of contradictory information questions the established division in pathogenicity of infertility between CFF and $\mathrm{CFV} / \mathrm{CFV} i$ as described above by the OIE and indicates that further research is needed in this regard.

One other problem in resolving issues between the subspecies is identification. Currently there are only a few phenotypic tests to differentiate CFF and CFV in most diagnostic laboratories. These include evaluation of growth on agar containing $1 \%$ glycine, generation of $\mathrm{H}_{2} \mathrm{~S}$ from cysteine rich media, and growth at $42^{\circ} \mathrm{C}$ (Wagenaar \& van der Graaf-van Bloois 2018). However these tests are poorly standardised, limited in number and complicated by CFVi strains, which are intermediate in their phenotype between CFF and CFV (van Bergen et al. 2008) particularly with respect to cysteine metabolism, a feature which appears to be conferred by an L-cysteine transporter (Farace et al. 2019; van der Graaf-van Bloois et al. 2016). One phenotypic characteristic, serotype analysis based on heat stable LPS antigens, has been fully characterized. Due to variation in both the genes ( $\operatorname{sap} A / \operatorname{sap} B)$ encoding surface array proteins and the surface lipopolysaccharide structure, CFF can be of serogroup A or B or AB (Dworkin et al. 1995) but CFV is always serogroup A. However, this has limited use since CFF strains are mostly serotype A. Overall, identification between subspecies using phenotypic and serological testing lacks either accuracy and/or reproducibility and, consequently, has equivocal usefulness.

Genetic based tools have been investigated in an effort to overcome this problem and to help correlate phenotypic and genomic characteristics of $C$. fetus subspecies (OIE 2018; van der Graaf-van Bloois et al. 2014). At the genomic level both pulse-field gel electrophoresis (PFGE) and amplified fragment length polymorphism (AFLP) have been reported to be useful methods for discriminating CFF and CFV (On \& Harrington 2001; Wagenaar et al. 2001) but the technicalities of these methods render them unsuitable for routine use in many laboratories. 
130 Multi-locus sequence typing (MLST), in which amplicons of seven housekeeping genes were

131

132

133

134

135

136

137

138

139

140

141

142

143

144

145

146

147

148

149

150

151

152

153

154

155

156

157

158

159

160

161

162

163

164

165

166

167 compared at the sequence level, identified several distinct types but overall these studies highlighted the clonal nature of $C$. fetus and failed to correlate sequence type with subspecies and biovar identity (van Bergen et al. 2005). Similarly rRNA gene loci were too highly conserved to be of value while sequences comprising the CRISPR-cas system, despite exhibiting significant variability, did not provide a useful means of $C$. fetus subspecies discrimination (Calleros et al. 2017). The suggestion that the two subspecies can be distinguished using a genomic island present only in CFV (Gorkiewicz et al. 2010) has not gained wide acceptance. Rapid and highly accurate methods involving amplification and detection of specific loci would be ideal if appropriate targets can be identified. To this end an early report targeted the carbon starvation gene (cst 1 ) for $C$. fetus detection and the parA gene, carried either chromosomally or on a plasmid, for the identification of CFV (Hum et al. 1997). Attempts to adapt the latter assay to a real-time format have been described (Chaban et al. 2012; McMillen et al. 2006) despite reports demonstrating the lack of specificity of this target (Schmidt et al. 2010; Spence et al. 2011). Meanwhile, alternative targets that perform more consistently include the $n a h E$ gene that encodes a sodium-hydrogen exchanger family protein for detection of all C. fetus while the ISCfe 1 transposase sequence is proposed as a specific marker for CFV, with the caveat that careful design of primers is needed for complete inclusivity of all samples carrying this sequence (McGoldrick et al. 2013; van der Graaf-van Bloois et al. 2013). Genetic targets that specifically identify CFF have not yet been reported.

In an effort to better understand other aspects associated with $\mathrm{CFF}$ versus $\mathrm{CFV}$, detailed comparisons of the genome organisation of CFF and CFV isolates have been undertaken using both draft and closed chromosome sequence information (Ali et al. 2012; Kienesberger et al. 2014; van der Graaf-van Bloois et al. 2014). These studies concluded that all mammalianassociated CFs share extensive genome synteny and vary primarily due to the presence of distinct pathogenicity islands dotted throughout the chromosome. While these studies clearly indicated the high concordance of the genome complement in all isolates, the organisation of this genetic information can be revealed only through comparison of closed genomes. Unfortunately, the closed genome comparisons reported to date have typically involved only one sub-species member in each case, and thus lack consideration of the range of genomic diversity within each sub-species. Challenges in generating complete, polished and annotated genomes for these organisms are due to their high AT content and large numbers of repetitive and mobile elements. As a result, most of the available genomic data for CF isolates is at the draft genome level only.

This report describes the use of multiple genomics methods and tools to generate closed genome sequences for seven phenotypically well characterised C. fetus isolates representative of the two mammalian-associated subspecies and their biovars. Comparison of these genomes with an additional seven complete and polished genomes recovered from the NCBI database illustrates that, with a few exceptions, all have a similar gene complement. However, their level of genome 
168

169

170

171

172

173

174

175

176

177

178

179

180

181

182

183

184

185

186

187

188

189

190

191

192

193

194

195

196

197

198

199

200

201

202

203

synteny varies enormously and mechanisms by which these changes may have occurred are explored.

These data add significantly to our understanding of the genomic variation within this bacterial species and should assist future studies to better understand its emergence and identify the specific features responsible for differences in tropism and pathogenicity. In addition, given the need to be able to accurately differentiate CFF from CFV/CFVi in cattle, these data may help, in practical terms, to identify useful additional markers for rapid and accurate subspeciation of isolates and provide a better understanding of the genes directly associated with BGC than currently exists.

\section{Materials and Methods}

Isolate growth and phenotypic characterization

C. fetus ssp. isolates analysed in this study (Table 1) originated from clinical specimens from cattle submitted for culture. Five of the isolates were cultured in Canada and were identified originally to subspecies using a full array of tests as described elsewhere (Devenish et al. 2005). The two C. fetus strains from outside Canada were identified to subspecies/biovar in the submitting laboratory and a record maintained in the stock culture collection. Upon identification internally or receipt externally, pure culture isolates were inoculated into vials of CryoStor cryopreservative solution (Innovatek Medical Inc., Vancouver, Canada) and frozen at $80^{\circ} \mathrm{C}$ as part of a larger stock culture collection. For routine verification of identification in this study, the seven frozen C. fetus ssp. strains were cultured on Cysteine Heart blood $(\mathrm{CH})$ agar or Mueller-Hinton agar containing 10\% sheep blood and incubated under microaerophilic conditions $\left(4 \% \mathrm{O}_{2}, 9.5 \% \mathrm{CO}_{2}, 86.5 \% \mathrm{~N}_{2}\right)$ for $2-4$ days at $36^{\circ} \mathrm{C}$. A highly specific $C$. fetus monoclonal based ELISA procedure was used for genus, species and serotype (A or B) identification of the isolates (Brooks et al. 2002; Wagenaar \& van der Graaf-van Bloois 2018).

For subspecies and biovar identification of the seven isolates, all media were inoculated with a prepared McFarland standard 1.0 suspension of the isolate, incubated under microaerophilic conditions at $36^{\circ} \mathrm{C}$ unless otherwise stated and tested for four different phenotypes as follows; 1) glycine tolerance - conducted in tubes of thioglycollate-135C broth supplemented with $0.0,0.6$, $1.0,1.3,1.5$ and $1.9 \%$ glycine into which $0.1 \mathrm{ml}$ of the bacterial suspension was inoculated. CFF isolates routinely grow at $1.3 \%$ glycine and above but never below, while CFV and CFVi isolates will grow at $1.0 \%$,or sometimes weakly at $1.1 \%$, and below but never at $\geq 1.3 \%$ glycine, 2) growth at $42^{\circ} \mathrm{C}$. A loopful of each isolate suspension was streaked onto two $\mathrm{CH}$ agar plates for well isolated colonies and incubated at $36^{\circ} \mathrm{C}$ or $42^{\circ} \mathrm{C}$ for 5 days. Using this procedure typically about $50 \%$ and $0.0 \%$ of CFF and CFV/CFVi isolates, respectively, show growth at the higher temperature of $\left.42^{\circ} \mathrm{C}, 3\right) \mathrm{H}_{2} \mathrm{~S}$ production-sensitive test. Brain heart infusion semi-solid 
204

205

206

207

208

209

210

211

212

213

214

215

216

217

218

219

220

221

222

223

224

225

226

227

228

229

230

231

232

233

234

235

236

237

238

239

240

medium with $0.022 \%$ L-cysteine was inoculated with $0.2 \mathrm{ml}$ of the standard suspension followed by addition of a lead acetate strip. Incubation of tubes was for 5 days after which they were observed for blackening of the lead acetate strip. Traditionally, $>95 \%$ of CFF and CFVi will be positive and show blackening of the lead acetate strip while $100 \%$ of CFV isolates will be negative, 4) ability to reduce selenite. A loopful of the bacterial strain immersed into sterile saline was streaked evenly over the surface of an Albimi agar slant containing $0.1 \%$ sodium selenite. The medium was incubated at $36^{\circ} \mathrm{C}$ for 5 days and observed for selenite reduction (reddening in the medium). Consistently $100 \%$ of CFF and CFVi isolates show reduction of the selenite and while approximately $20 \%$ of CFV isolates can be positive in this test procedure the reduction is much weaker. Known positive and negative control bacterial strains were included for comparison in all testing procedures.

DNA extraction

Colonies collected from agar plates were subjected to DNA extraction using a Wizard Genomic DNA extraction kit as per the supplier's directions (Promega) and quantification of the final solution was determined by a Qubit instrument using a broad sensitivity dsDNA quantification kit (Thermo Fisher Scientific, Waltham, MA, USA).

Optical mapping

Each $C$. fetus isolate was grown on plates prepared with Campylobacter agar base (Oxoid CM0689, Thermo Fisher) supplemented with 5\% laked horse blood (Oxoid SR0048) under 5\% $\mathrm{O}_{2}, 10 \% \mathrm{CO}_{2}$ at $37^{\circ} \mathrm{C}$ for two days. Single colonies were collected during the exponential growth phase for preparation of high molecular weight genomic DNA using an Argus DNA Extraction Kit. DNA was loaded on a MapCard following the manufacturer's instructions (OpGen, Inc, Gaithersburg, MD). After digestion with NcoI restriction enzyme, images of digested nucleotide molecules were captured and processed by an Argus Optical Mapping System (OpGen Inc.) equipped with a fluorescent microscope. A restriction map of a closed, circular genome was generated using Argus MapSolver ${ }^{\mathrm{TM}}$ software (OpGen Inc). Each optical map was compared to the in silico NcoI map generated from the assembled DNA sequence produced by whole genome sequencing data of the same isolate.

Whole genome sequencing

DNA extracts were subjected to multiple methods of whole genome sequencing. Pac Bio sequencing, performed by the McGill University and Genome Quebec Innovation Centre using PacBio RS II SMRT technology, generated between 45,000 to 107,000 raw reads, corresponding to 2 to $13 \times 10^{8}$ sequenced bases, per sample. Reads were error corrected and assembled using the Hierarchical Genome Assembly Process (HGAP) workflow (Ihon 2014) to generate between 1 to 12 contigs per isolate with average reference genome coverage between 75 and 495 . Contigs were ordered and oriented using the optical maps. The resulting draft genomes were used for reference based assemblies of paired end reads generated from indexed Nextera XT libraries run 
241 on an Illumina Hiseq 2000 sequencer $(2 \times 300$ except for CFF02A725 and CFV08A1102 which

242 were run earlier using 2 x 100 reagent kits) at the Michael Smith Genome Sciences Centre (BC,

243 Canada). Assemblies were initially generated and reviewed using the Lasergene Genomics suite

244 software v14 (DNASTAR Inc., Madison, WI) in an iterative process to fill gaps and remove

245 small errors introduced by the PacBio sequencing; Illumina reads added $>100$ average base

246 coverage. Small gaps in coverage for isolates CFF02A725 and CFV08A1102 were filled by

247 Sanger sequencing of PCR products. Final assemblies were confirmed by comparison with the

248 optical maps. To further confirm these genome assemblies and facilitate the assembly of any

249 plasmids harbored by these isolates long read sequencing was performed on barcoded samples

250 using a rapid sequencing kit run on a R9.4 MinION flow cell (Oxford Nanopore, Cambridge,

251 MA). The combined sequence data from all platforms (Illumina paired-end, Pac-Bio and

252 Nanopore reads) were assembled using Unicycler (Wick et al. 2017). Briefly, this software

253 employs the SPAdes tool for de novo assembly of the short-read data and then uses the long

254 reads to bridge gaps in these assemblies. The bridged assemblies then underwent multiple rounds

255 of short-read polishing.

256 Annotation and genome interrogation

257 Chromosomal and plasmid sequences were annotated using Prokka (Seemann 2014) with further 258 refinement using an in-house script (Duceppe 2019) that uses a clustering algorithm to improve

259 predicted annotation descriptions. Due to variation in annotation designations for some genes the

260 identification of comparable protein products in different isolates was, in some cases, performed

261 by cross searching several isolates with the predicted protein products. CRISPR loci were

262 identified using the web-based CRISPRFinder tool (Grissa et al. 2007). All genome alignments

263 were generated using the progressive Mauve option of Mauve version 2.3.1 (Darling et al. 2004)

264 using previously characterised sequences as reference. 


\section{Results}

266 Phenotypic analysis

267 This study compares fourteen representative mammalian-associated C. fetus isolates of which 12 268 originated from bovines while the remaining two CFFs are of human and ovine origin (Table 1).

269 Seven of these isolates, which are described in this report for the first time, were all identified as 270 C. fetus using a specific monoclonal antibody capture ELISA test and were found to possess 271 serotype A heat stable lipopolysaccharide (LPS) antigens. Details of their provenance (Table 1) 272 and the results of their phenotypic testing (Table S1) are presented. Based on the ability for 273 growth on media with $>1 \%$ glycine, generation of hydrogen sulphide in cysteine rich medium, 274 the ability to reduce selenite and grow at $42^{\circ} \mathrm{C}$, three isolates scored as positive in either three or 275 four tests and were identified as CFF. Two isolates which scored as negative by all four tests 276 were classified as CFV while the remaining two isolates were identified as CFVi based on 277 intermediate results that were shared with both CFV (inability to grow in broth with $>1 \%$ 278 glycine and at $42^{\circ} \mathrm{C}$ ) and $\mathrm{CFF}$ (production of $\mathrm{H}_{2} \mathrm{~S}$ and reduction of selenite). However, it is 279 evident from the results shown in Table S1 that a single biochemical test is sometimes 280 insufficient to yield an accurate sub-type classification. Phenotypic analysis supporting the 281 subspecies and biovar classification of the additional seven C. fetus isolates, as summarised in 282 Table 1, has been reported previously (van der Graaf-van Bloois et al. 2014). Since the focus of 283 this report was to compare bovine isolates of the different sub-species,

284 Genome sequencing and assembly

285

All seven newly characterised isolates were analysed by long (PacBio) and short (Illumina) read sequencing; all raw data, available in Genbank (Supplemental Table S2), was used for generation of closed chromosomal sequences as described. To assist with and confirm the accuracy of these assemblies each genomic sequence was used to generate a NcoI restriction map in silico for comparison with an experimentally generated optical map for each isolate (Supplemental Fig $\mathrm{S} 1$ ). These data indicate excellent concordance between both maps for all seven isolates, the nonaligned regions at the termini being a result of incomplete alignment of these circular chromosomes by a linear format. There was one small inconsistency in isolate CFF09A980 (Supplemental Fig S1) which could not be resolved, though the sizes of the fragments in this region of the genome did not appear to differ significantly between the optical map and the sequence read data. The later addition of MinIon sequence data (Supplemental Table S2) further confirmed these genome assemblies.

Genome analysis

\section{Genome organisation}

A summary of the characteristics of the closed circular chromosome of all fourteen $C$. fetus isolates is presented in Table 2. Overall the genome size appeared to reflect the subspecies and 
301

302

303

304

305

306

307

308

309

310

311

312

313

314

315

316

317

318

319

320

321

322

323

324

325

326

327

328

329

330

331

332

333

334

335

336

337

338

biovar classification with all CFF strains having the smallest genomes, of approximately $1.8 \mathrm{Mb}$, while the genomes of the CFV and CFVi strains were larger, generally between 1.9 and $2 \mathrm{Mb}$ with the exception of isolate CFVi03-293 which was a little smaller at $\sim 1.87 \mathrm{Mb}$. GC content was consistent at $33.2-33.3 \%$ except for two CFVi isolates which had a slightly higher content.

Chromosomal alignments were generated to compare the overall organisation of these $14 \mathrm{CF}$ genomes. Fig 1 shows a comparison of the overall genome organisation for three representative CFs, one each of CFF, CFV and CFVi while further comparisons between members of each subspecies and biovar are shown in Figs. 2-4. The alignment presented in Fig. 1 indicates an overall similar genome organisation for all three CFs but with two main observations: a hypervariable region, located variously between bases 450,000 to 600,000 , following a well conserved block of sequence and increasing length of the genome from CFF to CFV and CFVi members due to multiple insertions.

The six CFF isolates (Fig. 2) exhibited a high level of synteny with significant differences limited to a few relatively small regions. The sequences corresponding to residues 435,000 and 485,000 of the reference strain (CFF-04-554) were the most variable between all isolates and included significant rearrangements and gene inversions of blocks of sequence. There was also a variable sequence of $\sim 32 \mathrm{~kb}$ (corresponding to the white/pale orange block at bases 1,100,0001,113,200 of CFF04-554) which contained elements representative of a prophage that appeared in different locations in each isolate. Additional variations were observed in specific isolates including insertions of $45.4 \mathrm{~Kb}(1,203,385-1,248,755)$ in CFF-09A980 (described further below) and one of $\sim 30 \mathrm{~Kb}(650,000-680,000)$ in CFF 04-554 which encoded many hypothetical proteins and several different Fic protein alleles.

The alignment of five CFV genomes (Fig. 3) again identified a hypervariable region (corresponding to residues 532,000-616,675 of CFV-84-112) but it also revealed greater variability in other genomic regions amongst this group than was observed for the CFFs. Compared to the other CFVs, the CFV-84-112 genome included a transposition of a block of sequence of about $95 \mathrm{~Kb}$; the region affected (bases 142,200-237,000), which was bound by rRNA small and large subunit genes, corresponded to residues 544,240-638,200 of the other genomes. This genomic region encoded several membrane and periplasmic proteins, several of which had either signal transduction or transporter functions, including heavy metal transportation, products involved in heavy metal resistance and several enzymes involved in multiple metabolic pathways. A large genomic segment of CFV-84-112 corresponding to residues 761,700-1,275,130 was inverted compared to the other genomes. A $22 \mathrm{~Kb}$ sequence, representative of a genomic island harbouring Type IV secretion system genes (see below), located close to the downstream terminus was inverted in both CFV-97-608 and CFV-08A948 compared to the other three CFVs.

Yet additional variability was evident amongst the three CFVi isolates (Fig. 4). Indeed, when, as per convention, the $d n a A$ gene is placed at the start sequence, CFVi-ADRI1362 exhibited an 
339

340

341

342

343

344

345

346

347

348

349

350

351

352

353

354

355

356

357

358

359

360

361

362

363

364

365

366

367

368

369

370

371

372

373

374

375

376

inversion of a large section of the genome compared to the other CFVi isolates such that genome co-ordinates were significantly altered, though in reality this difference could be visualised as an inversion of a smaller genome segment of $\sim 405,000 \mathrm{bp}$. CFVi-ADRI545 exhibited a genome organisation closer to that of the reference genome but with several notable differences. The hypervariable region of this isolate was shifted downstream due to two insertions; indeed, this sample contained no less than six insertions relative to the reference as well as an inversion of residues 894,000-1,165,000 compared to the reference, a feature also shared by CFViADRI1362. The features contributing to these differences are detailed further below.

\section{Hypervariable region}

Annotation information for all 14 genomes facilitated comparison of their gene complement including a detailed comparison of the hypervariable region described above and recognised previously as a locus encompassing multiple sap gene alleles ( $\mathrm{Tu}$ et al. 2003). Comparison of the organisation of this region is shown in Fig 5 for 13 CFs, CFV-08A948 and CFV-08A1102 being virtually identical. This region includes two sets of gene groupings retained in virtually all isolates but varied in order and orientation. One set includes the genes tolC (an outer membrane protein), prs and prsD, which encode products involved in the Type 1 secretion system, and a presumed peptidase. Notably this gene group was absent in the single ovine isolate CFFNCTC10842 but it is unknown if this is typical of ovine isolates. The second set of 11 genes, present in all cases, is bound by the $s s r A$ gene encoding a tmRNA and the $y b e Y$ gene that encodes an endoribonuclease, except for CFV-84-112 in which the ssrA gene is missing. Between these two loci are genes encoding three transferases, a rhodanese, the acid membrane antigen $\mathrm{A}$ and four genes (mlaB, mlaD, mlaE and MlaF) all believed to be involved in lipid transport. In CFF-82-40 the mlaE locus is identified as a pseudo gene. Interspersed around these two gene groupings are multiple copies of the surface array protein genes, $\operatorname{sap} A$ for all 12 serogroup A isolates and sapB for the two serogroup B isolates, CFF-04-554 and CFFNCTC10842. Copy numbers of the sap genes range from six to 10. Additional genes found in this region for some isolates encode a mRNA interferase toxin (yafQ also known as relA), a hip A domain protein (hipA), an AAA family ATPase, additional surface associated products (sap) and some of unknown function. Indeed, the high proportion of genes that encode proteins involved in functions at the bacterial surface indicate the important pathogenic nature of this hypervariable region for all CFs.

\section{Selected gene complement}

Many of the genes of the hypervariable region, together with several additional genes presumed to be important with respect to virulence or phenotype, are detailed in Supplemental Table S3. In most cases these genes have been identified in all members of the Campylobacter genus (Ali et al. 2012) and are presumably necessary to support host cell adherence, invasion and immune evasion. As such in general they were retained in all $14 \mathrm{CFs}$ though with significant variation in location for some isolates. This included three copies of the cytolethal distending toxin operon 
377

378

379

380

381

382

383

384

385

386

387

388

389

390

391

392

393

394

395

396

397

398

399

400

401

402

403

404

405

406

407

408

409

410

411

412

413

comprised of three genes ( $c d t A, c d t B$ and $c d t C)$, with truncation of one or more alleles in some cases. In some isolates specific genes were lacking, including loci encoding a tyrosine recombinase $(x e r H)$, a filamentous hemagglutinin transporter protein $(f h a C)$ or the twitching mobility protein (pilT) but these variations did not respect the sub-species / biovar designations.

However, other coding differences did respect some of the observed phenotypic variation. All five $\mathrm{CFV}$ isolates lacked two genes ( $t c y A$ and $t c y C$ ) comprising the L-cys ABC transporter operon while the 3 '-terminal sequence of the remaining gene $(t c y B)$ was modified; all CFF and CFVi isolates retained the complete unaltered operon. Three genes previously described as significant with respect to CF variation in lipopolysaccharide biosynthesis (Kienesberger et al. 2014) were also examined. The glf locus that encodes a UDP-galactopyranose mutase was found in all serotype A CFFs but not in serotype B CFFs or any CFV/CFVi isolates. In contrast the mat 1 gene that encodes a maltose O-acetyltransferase activity was present in all isolates except for the serotype A CFFs. A $w c b K$ gene that encodes the enzyme GDP-mannose 4,6 dehydratase was found only in serotype B CFFs. Finally, it was noted that the transporter gene (annotated as $k e f C$ in this study) corresponding to the $n a h E$ target used previously for C. fetus detection was present in all isolates.

\section{Mobile genetic elements}

The presence of two groups of mobile genetic elements, transposons and prophages, throughout these $14 \mathrm{CF}$ genomes was highly variable (Table 3). Various copy numbers of two distinct versions of the IS200 family of insertion sequences, IS605 and IS607, were scattered throughout the genomes of all CFV and CFVi isolates and were frequently present in the hypervariable region. Indeed, while most of these isolates contained multiple copies of both types of transposon, CFV-84-112 contained two IS605 copies only and CFVi-ADRI1362 contained five copies of IS607 but no IS605. These elements were not present in any of the CFF genomes.

All CFs contained a prophage sequence ranging in length between 30 to $35 \mathrm{~Kb}$ but whereas the CFFs contained just one copy of this element all of the CFV and CFVi genomes, with the exception of CFV-NCTC10354 and CFVi-03-293, contained multiple copies; CFVi-ADRI1362 had no less than nine copies. This element was typically bound at either end by a copy of the lexA gene that encodes a prophage regulator, except for the prophage located in the hypervariable region of CFVi-ADRI1362 in which these loci were absent. The prophage sequence typically included genes encoding putative glutamine $\mathrm{ABC}$ transporter permease proteins, a mobile element, a modification methylase DpnIIA $(d p n M)$, capsid and tape measure domain-containing proteins, an ATPase, phage and portal proteins, the GP27 locus and many hypothetical products.

\section{CRISPR-cas complement}

The highly variable number of mobile elements present in these genomes led to a review of the presence of genes known to be involved in preventing invasion of the cell by foreign nucleic 
414

415

416

417

418

419

420

421

422

423

424

425

426

427

428

429

430

431

432

433

434

435

436

437

438

439

440

441

442

443

444

445

446

447

448

449

450

451

acids. This included a review of the components of the CRISPR-cas system. All 14 isolates contained at least one CRISPR locus and several of the CFFs contained two or more such loci (Table 4). Each locus contained 30 base direct repeats (DRs) of sequence GTTTGCTAATGACAATGTTTGTGTTGAAAC with occasional minor modification. Three isolates contained just one short locus of two (CFF-02A725), three (CFVi-03-293) and five (CFF-04-554) spacers respectively while the other isolates retained loci comprising 20-26 spacers. There was also significant variation in the presence of CRISPR-associated proteins. The four CFFs having two or more CRISPR loci encoded a complete set of cas genes (cas1 to cas6) while the other two CFFs retained a modified cas 6 gene only. All seven CFV/CFVi isolates also contained this modified cas 6 gene but lacked $\operatorname{cas} 1-5$ genes. A cas 9 pseudogene was identified in seven isolates representative of all three biotypes while this locus was not observed in the remaining isolates. With a few exceptions most other CRISPR-associated genes were retained though some had truncated ORFs.

\section{Restriction-modification system genes}

Review of the presence of restriction-modification (R-M) system genes, also capable of limiting invasion of the cell by foreign genetic material, indicated that most isolates retained a common but limited set of such genes though the serotype B CFFs were somewhat distinct in this regard due to a lack of several Type 1 R-M loci (Supplemental Table S3). However, review of the complement of so-called orphan methyltransferases that are unassociated with restriction endonucleases revealed some interesting differences. While all isolates retained one copy of an adenine-specific methyltransferase ( $f o k l$ ) all but one (CFF-04-554) contained one or more copies of a DNA adenine methylase (dam) gene. This gene appeared to be present as three distinct alleles which differ at their 5 ' termini and encode proteins ranging in length from 163 residues (allele 1) through 213 residues (allele 2) to 253 amino acids (allele 3). Alleles 1 and 2 were found only in CFF strains while between one to four copies of allele 3 were found in CFV and CFVi strains.

\section{Type IV secretion system genes}

In light of the significance made of the conservation of Type IV secretion system (Type IV SS) genes previously suggested for CFV isolates (Gorkiewicz et al. 2010), the presence of such genes in all CFs was investigated. Several distinct gene cassettes containing different combinations of genes of this group were identified (Table 5) and the structure of these genomic islands is illustrated (Fig 6). A genomic island (T4SS GI 1) of just under 40Kb containing several Type IV SS genes, including virB2 to virB11 and virD4, as well as genes believed to provide ancillary functions (Fic proteins and a lytic transglycosylase) and genes frequently plasmid-associated, such as $d n a G, t o p B$ and a tetracycline resistance ribosomal protection protein Tet(44), were identified in CFF-09A980. This sequence, corresponding to the insertion identified between $1,200,000$ and 1,250.000 in this isolate, was not present in any other CFF. A GI similar in composition and organisation was also found in CFV-84-112 and CFV-97-608 while CFVi03- 
452

453

454

455

456

457

458

459

460

461

462

463

464

465

466

467

468

469

470

471

472

473

474

475

476

477

478

479

480

481

482

483

484

485

486

487

488

489

490

293 and CFVi-ADRI545 retained a modified version of this GI in which some of the downstream genes were lost. Additional GIs harbouring a more limited group of these genes were also found. CFV-97-608 and CFV-84-112 harboured T4SS GIs 2 and 3 respectively while CFV-08A948 and CFV-08A1102 harboured only T4SS GI 4 which encompassed most core Type IV SS genes, $d n a \mathrm{G}$ as well as a DNA topoisomerase. Notably T4SS GIs 2 to 4 each incorporated an IS element likely explaining their transposition and modification. CFViADRI1362 contained only the T4SS GI 5 which harboured just four Type IV SS genes of highly modified sequence together with a topoisomerase, EcoRI methylase and mobC genes.

\section{Plasmid sequences and annotation}

The hybrid assembly process, which also included MinION sequence data, permitted the identification and assembly of plasmid-like elements found in six of the seven isolates characterised in this study. Of the complete collection of 14 isolates, three CFF strains appeared to lack these elements, the other three CFFs each contained a single plasmid and the CFV and CFVi isolates harboured between one to four plasmids each (Table 6). Annotation summaries of these plasmids is provided (Supplemental Table S4).

The genetic complement of these plasmids generally included multiple genes encoding conjugal transfer proteins of the Tra and Trb families that are involved in plasmid replication and in most cases several genes of the Type IV secretion system. Except for CFVi-03-293, at least one copy of the parA gene (designated as soj in our annotations), which encodes a plasmid partitioning protein, was found in all plasmid-harboring isolates, including the CFFs. This observation reinforces prior findings that this gene is not a specific marker for bovine-adapted strains. Genes encoding various toxin-antitoxin products $(y a f Q, \operatorname{vap} C)$ and a phage repressor protein of the BRO family were present on plasmids in all 11 isolates except for CFF-04-554 (yafQ, BRO), CFV-08A948 (BRO) and CFVi-03-293 (BRO). CFV and CFVi isolates harboured additional genes including Fic proteins (except CFV-84-112), relaxases and helicases, lytic transglycosylases, transcriptional regulators, as well as IS605 and IS607 elements which were often present in multiple copies. The plasmids of CFVi-ADRI1362 encoded several additional products that appear to have been obtained from a variety of different proteobacteria, including additional toxins, various enzymatic activities, ABC transporter components, a phage antirepressor, the cag pathogenicity island protein, cpp46 and copg family domain proteins. The P3 plasmid of CFVi-ADRI1362 and the P2 plasmid of CFVi-03-293 encoded essentially the same products, including a Type IV SS operon, virtually complete except for a short internal segment. Both strains also contained the same cryptic plasmid of 3,993 bp despite some differences in the predicted coding capacity of this sequence. The remaining plasmids were however quite distinct. CFVi-ADRI1362-P1 harboured several copies of IS605, an element which is absent from the chromosome of this isolate (Table 3 ). The high genetic plasticity provided by these extrachromosomal elements was especially evident for the two CFV isolates, CFV-08A948 and CFV-08A1102, both collected in the province of Alberta, Canada, in 2008 from separate AI facilities. The chromosomes of these two isolates were highly clonal thus suggesting a common 
491

492

493

494

495

496

497

498

499

500

501

502

503

504

505

506

507

508

509

510

511

512

513

514

515

516

517

518

519

520

521

522

523

524

525

526

source of infection. However, their plasmids did exhibit some coding differences. CFV08A948P1 contained two copies of a rha family transcriptional regulator and a $t r b E$ gene not found in CFV-08A1102P1 as well as one additional IS605 transposon. Products encoded by CFV-08A1102P1 but not CFV-08A948P1 included a BRO phage repressor protein, a single stranded binding protein and an adenosine monophosphate protein transferase $(v b h T)$. In addition to these differences some gene products, including those of $\operatorname{tr} b F$, $\operatorname{tr} b L$ and $\operatorname{tr} b J$, exhibited significant sequence differences.

\section{Discussion}

Many studies have identified the clonal nature of $C$. fetus isolates (van Bergen et al. 2005), a fact that has prompted studies on these organisms to better understand the genetic basis for their variable phenotype and host tropism. Phylogenetic analysis of MLST data has revealed the evolution of $C$. fetus, beginning with the emergence of distinct lineages normally associated with reptilian and mammalian hosts respectively, followed by division of the mammalian lineage into two serotypes, A and B, and the later emergence of the CFV subspecies from the A serotype (Dingle et al. 2010; Wang et al. 2013).

The studies presented in this report and by others (Kienesberger et al. 2014; van der Graaf-van Bloois et al. 2016) strongly suggest that the recent emergence of the C.fetus venerealis spp. occurred in parallel with increased genome plasticity associated with the acquisition, through horizontal gene transmission (HGT), of multiple mobile elements including members of the IS200 family of transposons, prophages and plasmid-like extrachromosomal elements.

Moreover, the greater genome plasticity exhibited by the CFVi isolates does suggest that these isolates represent a distinct group.

While differences in the presence or absence of these elements can be scored using draft genome sequence data, their context can be determined only through the examination of closed, polished genomes. Relatively few studies on $C$. fetus genomic structure have used such data for subspecies comparison and those reports have generally included very few (often just two) isolates (Ali et al. 2012; Kienesberger et al. 2014; van der Graaf-van Bloois et al. 2014; van der Graaf-van Bloois et al. 2016). This report, which is the first to compare the organisation and gene complement of a significant number of complete closed C. fetus genomes, provides additional insights into the role that different elements may have played in the evolution of these genomes, particularly with respect to the emergence of CFV and CFVi.

Of the two types of mobile elements found in the CF chromosome, only CFV/CFVi strains harbored members of the IS200 transposon family, often in multiple copies as previously reported (van der Graaf-van Bloois et al. 2013), and thus the ISCfel element has become a target for development of sub-species discriminatory PCR-based tools (Abril et al. 2007; McGoldrick 
527 et al. 2013). Clearly the spread of these elements throughout the genome raises the possibility of

528

529

530

531

532

533

534

535

536

537

538

539

540

541

542

543

544

545

546

547

548

549

550

551

552

553

554

555

556

557

558

559

560

561

562

563

564

565 gene disruption and function loss though it is notable that many of these elements were not located at points of genome rearrangement and thus may not be a primary driver of large-scale genome plasticity.

In contrast all CF isolates examined in this report contained prophage sequence. While the CFFs contained just one copy of this element, CFV/CFVi strains tended to harbour greater copy numbers of these elements and very notably these prophage sequences were often located at the boundaries of sequence rearrangements and inversions supporting the importance of this element type to genome plasticity. The potential role of prophage mobility in altering the gene complement is clearly illustrated by the sequence of CFF-02A725 in which prophage translocation (residues 1,127,063-1,160,613) resulted in the loss of several components of the CRISPR-cas system. The presence of prophage sequence in the hypervariable region of CFV and CFVi isolates may well have contributed to its evolution though its absence in that region of the CFFs suggests that other factors, including possibly homologous recombination involving the sap gene loci, may have contributed to the plasticity of this region (Tu et al. 2003). Indeed, the identification of rare recombinants having an $\mathrm{AB}$ serotype supports the high plasticity of this genomic region (Dingle et al. 2010).

Bacteria have a number of mechanisms to limit the extent to which foreign genetic material can invade a cell and become incorporated into the chromosome. Restriction-modification systems of many bacteria facilitate degradation of invading sequences having specific sequence motifs; however, C. fetus appears to have limited capability in this regard with no clear distinction detected between CFF and CFV subspecies. Another important process involves the CRISPR-cas system that is believed to confer an adaptive immune response to protect against invasion by mobile genetic elements (van der Oost et al. 2014). The CRISPR locus consists of a series of repeat sequences interspersed with spacer sequences derived from invading genetic material. Cas proteins are responsible for acquisition of these spacers as well as processing of RNA transcripts of these loci in a process that ultimately results in the degradation of DNA homologous to the spacer sequence. Three distinct CRISPR-cas systems with different mechanisms of action have been identified but in each case the cas 1 and cas 2 genes are critical to the initial steps of the process that incorporate the spacer sequences into the CRISPR locus and that later serve as templates for subsequent recognition of matched sequence elements. Cas 6 and cas 9 genes act as nucleases which process CRISPR transcripts in preparation for assembly into ribonucleoprotein particles used for surveillance and ultimately degradation of foreign DNA complementary to a spacer sequence (van der Oost et al. 2014). Variation in the CRISPR-cas system complement of C. fetus had been reported previously (Gilbert et al. 2016; van der Graaf-van Bloois et al. 2014) and this was further examined in this study. Four CFFs had two or more CRISPR loci and a virtually complete complement of cas and CRISPR-associated genes representative of a functional CRISPR-cas system. Two CFFs, CFF-04-554 and CFF-02A725, as well as all CFV and CFVi isolates lacked cas 1 -cas 6 genes but retained a modified cas6, cas 10 , a member of the

Peer) reviewing PDF | (2020:06:49901:2:0:NEW 23 Nov 2020) 
566 Type III CRISPR-cas system, and some other CRISPR-related genes. While these isolates may

567 retain the ability to process spacer transcripts and thereby prevent invasion of elements

568 containing these sequences, the loss of cas 1 and cas 2 would preclude addition of sequences to

569 the CRISPR locus and thereby limit the scope of the system. In those isolates in which the spacer

570 number is significantly reduced the value of this system is clearly highly compromised.

571 Limitations in the functioning of the CRISPR-cas system could provide various prophage and

572 transposable elements the opportunity to successfully invade the cell and integrate into the

573 chromosome. While this in itself would not explain the phenotypic changes that have

574 accompanied the emergence of the CFV/CFVi strains, the possibility of random mobile element

575 insertion into the bacterial chromosome and spread within the genome could result in loss of

576 function due to gene deletion or changes in gene expression as well as increased genome

577 instability. Consistent with our observations, it has been reported that the screening of a

578 collection of 102 C. fetus isolates for the presence of cas 1 failed to detect this gene in all $62 \mathrm{CFV}$

579 samples (Kienesberger et al. 2014) and it was later suggested that disruption to the CRISPR-cas

580 system could be a significant factor contributing to the emergence of CFV/CFVi strains (Calleros

581 et al. 2017). We speculate that similar loss of CRISPR-cas functionality in CFF isolates, as found

582 here, might initiate a series of genomic modifications to alter their pathogenicity, thus explaining

583 their potential ability to produce infertility in cattle as discussed above. Clearly more work would

584 be needed to substantiate this possibility, but if true, may further blur the distinction between

585 these subspecies in pathogenicity and the role of the two subspecies in BGC.

586 One notable distinction found between CFF and CFV/CFVi isolates concerns differences with

587 respect to their predicted dam gene complement. This gene, first identified and extensively

588 characterised in Escherichia coli, encodes DNA adenine methylase responsible for post-

589 replicative adenine methylation at GATC sites along the genome. DNA methylation is an

590 important epigenetic process that can impact aspects of DNA replication, including mismatch

591 repair, as well as gene expression (Adhikari \& Curtis 2016; Casadesús \& Low 2006). Distinct

592 alleles of a gene predicted to have this activity were found in CFF and CFV/CFVi strains;

593 furthermore, multiple copies of allele 3 of this gene were present in six of the eight CFV/CFVi

594 isolates examined. The structural differences between these dam products and potential variation

595 in their expression levels due to variable gene copy number could have significant impact on the

596 extent of GATC methylation of these genomes and, in turn, effect differences in expression of

597 other genes. This aspect of $C$. fetus biology is worthy of further investigation considering that

598 dam methylation is believed to impact host-pathogen interactions through modification of

599 virulence gene expression of several bacteria (Marinus \& Casadesus 2009).

600 This report has also explored the plasmid composition of these $14 \mathrm{CF}$ strains as these

601 extrachromosomal elements can contribute genes that impart additional phenotypic features to

602 the organism. Greater numbers of these elements were identified in CFV and particularly CFVi

603 strains, again supporting the concept that these strains are deficient in their ability to limit

604 cellular invasion by foreign DNA. Apart from genes important for replication of these elements, 
605 Type IV secretory system genes were the most common. Indeed, a complete T4SS operon was

606 found in CFVi-ADRI1362 P3, thereby complementing the limited gene set present in the

607 chromosome. These genes have undoubtedly been acquired through horizontal transmission from

608 other bacteria (Kienesberger et al. 2014) with varying levels of incorporation into the CF

609 chromosome. Moreover, in contrast to prior claims that Type IV secretion system genes were

610 restricted to CFV/CFVi strains, a complete T4SS GI, which corresponds closely to that described

611 previously (Gorkiewicz et al. 2010), was found in one of our CFF genomes (CFF-09A980).

612 While these genes are clearly implicated in virulence and pathogenicity their variable

613 complement across these CFs suggests that these functions are likely not restricted to one sub-

614 species.

615 Critical to this analysis was the comprehensive phenotypic designation of all seven newly

616 analysed isolates described in this study together with their accurate and complete genome

617 characterisation using data derived from multiple short and long read sequencing methods

618 combined with optical mapping for confirmation of genome assembly. The additional seven

619 isolates used for comparison here have also been extensively studied previously, both genetically

620 and phenotypically. These data permitted an evaluation of specific genes identified as either

621 conferring phenotypic differences between the subspecies and / or having utility for subspecies

622 differentiation. The value of the ISfe target for detection of CFV/CFVi strains has already been

623 discussed above. The ability to generate $\mathrm{H}_{2} \mathrm{~S}$ has been correlated with presence of the L-cys

624 transporter operon comprised of three genes ( $t c y A, t c y B$ and $t c y C$ ) responsible for production of a

625 functional cysteine transporter complex. It was found that all CFV isolates did indeed lack two of

626 these genes while they were retained in all CFVi and CFF isolates. Notably the CFF-00A031

627 isolate which had been scored as $\mathrm{H}_{2} \mathrm{~S}$ negative in this study harbored all three genes and would

628 appear to have a functional transporter complex though this has not been verified by expression

629 studies. This observation underscores the challenges in phenotypic evaluation of these highly

630 fastidious organisms and highlights the need for improved genomics-based classification. Indeed

631 it is noteworthy that the CFVi-ADRI1362 isolate has previously been reported as having a CFF

632 phenotype but a genome consistent with its correct designation as described here (van der Graaf-

633 van Bloois et al. 2014). A study exploring the enzymes involved in LPS biosynthesis identified

634 three genes ( $g l f$, matl and $w c b K$ ) which collectively showed good correlation with $C$. fetus

635 subspecies with serotype A CFFs scoring as $\mathrm{glf}^{+}$, mat $^{-}$, wcbK ${ }^{-}$, serotype B CFFs as glf $^{-}$, mat $^{+}$,

$636 w_{c b K^{+}}$and all CFVs as $g l f^{-}, \mathrm{mat}^{+}, w c b K^{-}$(Kienesberger et al. 2014). Our analysis of $14 \mathrm{CFs}$ is

637 entirely consistent with this genotype scheme. It has been suggested that the $w c b K$ gene product

638 may aid bacterial cell viability in low $\mathrm{pH}$ environments and thus facilitate intestinal colonization

639 following ingestion; its absence in all CFVs might in part explain why these strains are most

640 often recovered from the genital tract; however this does not explain how serotype A CFFs

641 navigate the stomach environment. These genetic targets might be further evaluated for their

642 utility in accurate subspecies and biovar identification. As a better understanding of the genetic

643 basis that underpins the distinct pathogenicity of CF isolates is achieved, genetic methods for

644 their discrimination will provide for more accurate and meaningful BGC diagnosis and control.

Peer] reviewing PDF | (2020:06:49901:2:0:NEW 23 Nov 2020) 


\section{Conclusion}

646 Our studies overall suggest that $C$. fetus isolates, even those classified as CFF, harbour 647 chromosomes which exhibit some variation in their gene complement and, apart from the 648 retention of a hypervariable region, pathogenicity islands previously described in other studies 649 are not preserved even at the subspecies level. Many intermediate genetic types have evolved as 650 a result of ongoing mobile element acquisition and insertion, genome rearrangement and 651 acquisition or loss of specific genes that collectively may contribute to the variable pathologies 652 caused by these organisms. Variation in the gene complement of extrachromosomal elements 653 further complicates the situation. Deposition of increasing numbers of polished genomes to 654 public databases will help to further clarify the classification of this species and fully understand 655 the genetic basis for its variable pathogenesis.

656

\section{Acknowledgements}

658 We thank Greg Appleyard for his preliminary studies on the genetic differences between C. fetus 659 strains that stimulated this study. We also thank Mary Sheen and Teresa Burke for excellent 660 technical support.

661 
662

663

664

665

666

667

668

669

670

671

672

673

674

675

676

677

678

679

680

681

682

683

684

685

686

687

688

689

690

691

692

693

694

695

696

697

698

699

700

701

702

703

\section{References}

Abril C, Vilei EM, Brodard I, Burnens A, Frey J, and Miserez R. 2007. Discovery of insertion element ISCfe1: a new tool for Campylobacter fetus subspecies differentiation. Clinical Microbiology and Infection 13:993-1000. 10.1111/j.1469-0691.2007.01787.x

Adhikari S, and Curtis PD. 2016. DNA methyltransferases and epigenetic regulation in bacteria. FEMS Microbiology Reviews 40:575-591. 10.1093/femsre/fuw023

Ali A, Soares SC, Santos AR, Guimarães LC, Barbosa E, Almeida SS, Abreu VAC, Carneiro AR, Ramos RTJ, Bakhtiar SM, Hassan SS, Ussery DW, On S, Silva A, Schneider MP, Lage AP, Miyoshi A, and Azevedo V. 2012. Campylobacter fetus subspecies: Comparative genomics and prediction of potential virulence targets. Gene 508:145-156. https://doi.org/10.1016/j.gene.2012.07.070

Brooks BW, Robertson RH, Lutze-Wallace CL, and Pfahler W. 2002. Monoclonal antibodies specific for Campylobacter fetus lipopolysaccharides. Veterinary Microbiology 87:37-49.

Butzler J-P. 2004. Campylobacter, from obscurity to celebrity. Clinical Microbiology and Infection 10:868-876. 10.1111/j.1469-0691.2004.00983.x

Calleros L, Betancor L, Iraola G, Méndez A, Morsella C, Paolicchi F, Silveyra S, Velilla A, and Pérez R. 2017. Assessing the intra-species genetic variability in the clonal pathogen Campylobacter fetus: CRISPRs are highly polymorphic DNA markers. Journal of Microbiological Methods 132:86-94. https://doi.org/10.1016/j.mimet.2016.11.012

Casadesús J, and Low D. 2006. Epigenetic Gene Regulation in the Bacterial World. Microbiology and Molecular Biology Reviews 70:830-856. 10.1128/mmbr.00016-06

Chaban B, Chu S, Hendrick S, Waldner C, and Hill JE. 2012. Evaluation of a Campylobacter fetus subspecies venerealis real-time quantitative polymerase chain reaction for direct analysis of bovine preputial samples. The Canadian Journal of Veterinary Research 76:166-173.

Clark BL. 1971. Review of bovine vibriosis. Australian Veterinary Journal 47:103-107. https://doi.org/10.1111/j.1751-0813.1971.tb14749.x

Darling ACE, Mau B, Blattner FR, and Perna NT. 2004. Mauve: Multiple Alignment of Conserved Genomic Sequence With Rearrangements. Genome Research 14:1394-1403. 10.1101/gr.2289704

Dekeyser J. 1984. Bovine Genital Campylobacteriosis. In: Butzler JP, ed. Campylobacter Infection in Man and Animals. Boca Raton, Florida: CRC Press, 181-192.

Devenish J, Brooks B, Perry K, Milnes D, Burke T, McCabe D, Duff S, and Lutze-Wallace CL. 2005. Validation of a Monoclonal Antibody-Based Capture Enzyme-Linked Immunosorbent Assay for Detection of Campylobacter fetus. Clinical and Diagnostic Laboratory Immunology 12:1261-1268.

Dingle KE, Blaser MJ, Tu Z-C, Pruckler J, Fitzgerald C, van Bergen MAP, Lawson AJ, Owen RJ, and Wagenaar JA. 2010. Genetic Relationships among Reptilian and Mammalian Campylobacter fetus Strains Determined by Multilocus Sequence Typing. Journal of Clinical Microbiology 48:977-980. 10.1128/jcm.01439-09

Duceppe M-O. 2019. Bacterial genome assembly. Available at https://github.com/duceppemo/bacteria genome assembly/blob/master/bda.py. 
704

705

706

707

708

709

710

711

712

713

714

715

716

717

718

719

720

721

722

723

724

725

726

727

728

729

730

731

732

733

734

735

736

737

738

739

740

741

742

743

744

745

746

747

748
Dworkin J, Tummuru MKR, and Blaser MJ. 1995. Segmental Conservation of sapA Sequences in Type B Campylobacter fetus Cells. Journal of Biological Chemistry 270:15093-15101. 10.1074/jbc.270.25.15093

Farace PD, Morsella CG, Cravero SL, Sioya BA, Amadio AF, Paolicchi FA, and Gioffré AK. 2019. L-cysteine transporter-PCR to detect hydrogen sulfide-producing Campylobacter fetus. PeerJ 7:e7820. 10.7717/peerj.7820

Fitzgerald C. 2015. Campylobacter. Clinics in Laboratory Medicine 35:289-298. https://doi.org/10.1016/j.cll.2015.03.001

Fitzgerald C, Tu Zc, Patrick M, Stiles T, Lawson AJ, Santovenia M, Gilbert MJ, van Bergen M, Joyce K, Pruckler J, Stroika S, Duim B, Miller WG, Loparev V, Sinnige JC, Fields PI, Tauxe RV, Blaser MJ, and Wagenaar JA. 2014. Campylobacter fetus subsp. testudinum subsp. nov., isolated from humans and reptiles. International Journal of Systematic and Evolutionary Microbiology 64:2944-2948. https://doi.org/10.1099/ijs.0.057778-0

Florent A. 1963. A propos dese vibrions responsables de la vibriose génitale des bovins et des ovins. Bulletin de l'Office International des Épizooties 60:1063-1074.

Gazaigne L, Legrand P, Renaud B, Bourra B, Taillandier E, Brun-Buisson C, and Lesprit P. 2008. Campylobacter fetus bloodstream infection: risk factors and clinical features. European Journal of Clinical Microbiology \& Infectious Diseases 27:185-189. 10.1007/s10096-007-0415-0

Gilbert MJ, Miller WG, Yee E, Zomer AL, van der Graaf-van Bloois L, Fitzgerald C, Forbes KJ, Méric G, Sheppard SK, Wagenaar JA, and Duim B. 2016. Comparative Genomics of Campylobacter fetus from Reptiles and Mammals Reveals Divergent Evolution in HostAssociated Lineages. Genome Biology and Evolution 8:2006-2019. 10.1093/gbe/evw146

Gorkiewicz G, Kienesberger S, Schober C, Scheicher SR, Gülly C, Zechner R, and Zechner EL. 2010. A Genomic Island Defines Subspecies-Specific Virulence Features of the HostAdapted Pathogen Campylobacter fetus subsp. venerealis. Journal of Bacteriology 192:502-517. 10.1128/jb.00803-09

Grissa I, Vergnaud G, and Pourcel C. 2007. CRISPRFinder: a web tool to identify clustered regularly interspaced short palindromic repeats. Nucleic Acids Research 35:W52-W57. 10.1093/nar/gkm360

Hum S, Quinn K, Brunner J, and On SL. 1997. Evaluation of a PCR assay for identification and differentiation of Campylobacter fetus subspecies. . Australian Veterinary Journal 75:827-831.

Ihon. 2014. HGAP in SMRT Analysis. Available at https://github.com/PacificBiosciences/Bioinformatics-Training/wiki/HGAP-in-SMRTAnalysis (accessed November 2017.

Kienesberger S, Sprenger H, Wolfgruber S, Halwachs B, Thallinger G, Perez-Perez G, Blaser M, Zechner E, and Gorkiewicz G. 2014. Comparative genome analysis of Campylobacter fetus subspecies revealed horizontally acquired genetic elements important for virulence and niche specificity. PLoS One 9:e85491.

Marinus MG, and Casadesus J. 2009. Roles of DNA adenine methylation in host-pathogen interactions: mismatch repair, transcriptional regulation, and more. FEMS Microbiology Reviews 33:488-503. 10.1111/j.1574-6976.2008.00159.x

McGoldrick A, Chanter J, Gale S, Parr J, Toszeghy M, and Line K. 2013. Real Time PCR to detect and differentiate Campylobacter fetus subspecies fetus and Campylobacter fetus

Peer] reviewing PDF | (2020:06:49901:2:0:NEW 23 Nov 2020) 
subspecies venerealis. Journal of Microbiological Methods 94:199-204.

https://doi.org/10.1016/j.mimet.2013.06.014

McMillen L, Fordyce G, Doogan VJ, and Lew AE. 2006. Comparison of Culture and a Novel 5' Taq Nuclease Assay for Direct Detection of Campylobacter fetus subsp. venerealis in Clinical Specimens from Cattle. Journal of Clinical Microbiology 44:938-945. $10.1128 / \mathrm{jcm} .44 .3 .938-945.2006$

OIE. 2018. Bovine Genital Campylobacteriosis. Manual of Diagnostic Tests and Vaccines for Terrestrial Animals. 8th ed. Paris: Office International des Epizooties. p 1031-1044.

On SLW, and Harrington CS. 2001. Evaluation of numerical analysis of PFGE-DNA profiles for differentiating Campylobacter fetus subspecies by comparison with phenotypic, PCR and 16S rDNA sequencing methods. Journal of Applied Microbiology 90:285-293. 10.1046/j.1365-2672.2001.01247.x

Pacanowski J, Lalande V, Lacombe K, Boudraa C, Lesprit P, Legrand P, Trystram D, Kassis N, Arlet G, Mainardi J-L, Doucet-Populaire F, Girard P-M, Meynard J-L, and Group CS. 2008. Campylobacter Bacteremia: Clinical Features and Factors Associated with Fatal Outcome. Clinical Infectious Diseases 47:790-796. 10.1086/591530

Park R, Munro I, Melrose D, and Stewart D. 1962. Observations on the ability of two biochemical types of Vibrio fetus to proliferate in the genital tract of cattle and their importance with resepct to infertility. British Veterinary Journal 118:411.

Parte AC. 2018. LPSN - List of Prokaryotic names with Standing in Nomenclature (bacterio.net), 20 years on. International Journal of Systematic and Evolutionary Microbiology 68:1825-1829. https://doi.org/10.1099/ijsem.0.002786

Schmidt T, Venter EH, and Picard JA. 2010. Evaluation of PCR assays for the detection of Campylobacter fetus in bovine preputial scrapings and the identification of subspecies in South African field isolates. Journal of the South African veterinary Association 81:8792.

Seemann T. 2014. Prokka: rapid prokaryotic genome annotation. Bioinformatics 30:2068-2069. 10.1093/bioinformatics/btu153

Silva MF, Pereira G, Carneiro C, Hemphill A, Mateus L, Lopes-da-Costa L, and Silva E. 2020. Campylobacter portucalensis sp. nov., a new species of Campylobacter isolated from the preputial mucosa of bulls. PLoS One 15:e0227500. 10.1371/journal.pone.0227500

Silveira CdS, Fraga M, Giannitti F, Macías-Rioseco M, and Riet-Correa F. 2018. Diagnosis of Bovine Genital Campylobacteriosis in South America. Frontiers in Veterinary Science 5. 10.3389/fvets.2018.00321

Spence RP, Bruce IR, McFadden AMJ, Hill FI, Tisdall D, Humphrey S, van der Graaf L, van Bergen MAP, and Wagenaar J. 2011. Cross-reaction of a Campylobacter fetus subspecies venerealis real-time PCR. Veterinary Record 168:131.

Sprenger H, Zechner E, and Gorkiewicz G. 2012. So close and yet so far - molecular microbiology of Campylobacter fetus subspecies. European Journal of Microbiology and Immunology 2:66-75.

Tu Z-C, Wassenaar TM, Thompson SA, and Blaser MJ. 2003. Structure and genotypic plasticity of the Campylobacter fetus sap locus. Molecular Microbiology 48:685-698. 10.1046/j.1365-2958.2003.03463.x

van Bergen M, van Putten J, Dingle K, Blaser M, and Wagenaar J. 2008. Isolation, identification, subspecies differentiation, and typing of Campylobacter fetus. In: Nachamkin I, 
794

795

796

797

798

799

800

801

802

803

804

805

806

807

808

809

810

811

812

813

814

815

816

817

818

819

820

821

822

823

824

825

826

827

828

829

830

831

832

833

834

835

836

837

838

Szymanski C, and Blaser M, eds. Campylobacter. Washington, DC: ASM Press, 213225.

van Bergen MAP, Dingle KE, Maiden MCJ, Newell DG, van der Graaf-Van Bloois L, van Putten JPM, and Wagenaar JA. 2005. Clonal Nature of Campylobacter fetus as Defined by Multilocus Sequence Typing. Journal of Clinical Microbiology 43:5888-5898. $10.1128 / \mathrm{jcm} .43 .12 .5888-5898.2005$

van der Graaf-van Bloois L, Miller W, Yee E, Rijnsburger M, Wagenaar J, and Duim B. 2014. Inconsistency of phenotypic and genomic characterisatics of Campylobacter fetus subspecies requires reevaluation of current diagnostics. Journal of Clinical Microbiology 52:4183-4188.

van der Graaf-van Bloois L, van Bergen MAP, van der Wal FJ, de Boer AG, Duim B, Schmidt T, and Wagenaar JA. 2013. Evaluation of molecular assays for identification Campylobacter fetus species and subspecies and development of a C. fetus specific realtime PCR assay. Journal of Microbiological Methods 95:93-97. https://doi.org/10.1016/j.mimet.2013.06.005

van der Graaf-van Bloois L, Duim B, Miller WG, Forbes KJ, Wagenaar JA, and Zomer A. 2016. Whole genome sequence analysis indicates recent diversification of mammal-associated Campylobacter fetus and implicates a genetic factor associated with $\mathrm{H} 2 \mathrm{~S}$ production. BMC Genomics 17:713. 10.1186/s12864-016-3058-7

van der Oost J, Westra ER, Jackson RN, and Wiedenheft B. 2014. Unravelling the structural and mechanistic basis of CRISPR-Cas systems. Nature Reviews Microbiology 12:479-492. $10.1038 /$ nrmicro3279

Veron M, and Chatelain R. 1973. Taxonomy study of the genus Campylobacter Sebald and Veron and designation of the neotype strain for the type species, Campylobacter fetus (Smith and Taylor) Sebald and Veron. International Journal of Systematic Bacteriology 23:122-134.

Wagenaar JA, van Bergen MAP, Blaser MJ, Tauxe RV, Newell DG, and van Putten JPM. 2014. Campylobacter fetus Infections in Humans: Exposure and Disease. Clinical Infectious Diseases 58:1579-1586. 10.1093/cid/ciu085

Wagenaar JA, van Bergen MAP, Newell DG, Grogono-Thomas R, and Duim B. 2001. Comparative Study Using Amplified Fragment Length Polymorphism Fingerprinting, PCR Genotyping, and Phenotyping To Differentiate Campylobacter fetus Strains Isolated from Animals. Journal of Clinical Microbiology 39:2283-2286. 10.1128/jcm.39.6.22832286.2001

Wagenaar JA, and van der Graaf-van Bloois L. 2018. Bovine Genital Campylobacteriosis. Manual of Diagnostic Tests and Vaccines for Terrestrial Animals. 8th ed. Paris, France: OIE World Organization for Animal Health, 1031-1044.

Wang C-M, Shia W-Y, Jhou Y-J, and Shyu C-L. 2013. Occurrence and molecular characterization of reptilian Campylobacter fetus strains isolated in Taiwan. Veterinary Microbiology 164:67-76. https://doi.org/10.1016/j.vetmic.2013.01.008

Wick RR, Judd LM, Gorrie CL, and Holt KE. 2017. Unicycler: Resolving bacterial genome assemblies from short and long sequencing reads. PLOS Computational Biology 13:e1005595. 10.1371/journal.pcbi.1005595

Woo PC-Y, Leung K-W, Tsoi H-W, Wong SS-Y, Teng JL-L, and Yuen K-Y. 2002. Thermotolerant Campylobacter fetus bacteraemia identified by 16S ribosomal RNA gene 
Figure 1

Alignment of three CF genomes representative of CFF, CFV and CFVi

Assembled genomes were aligned using the progressive Mauve option of Mauve software v2.3.1.

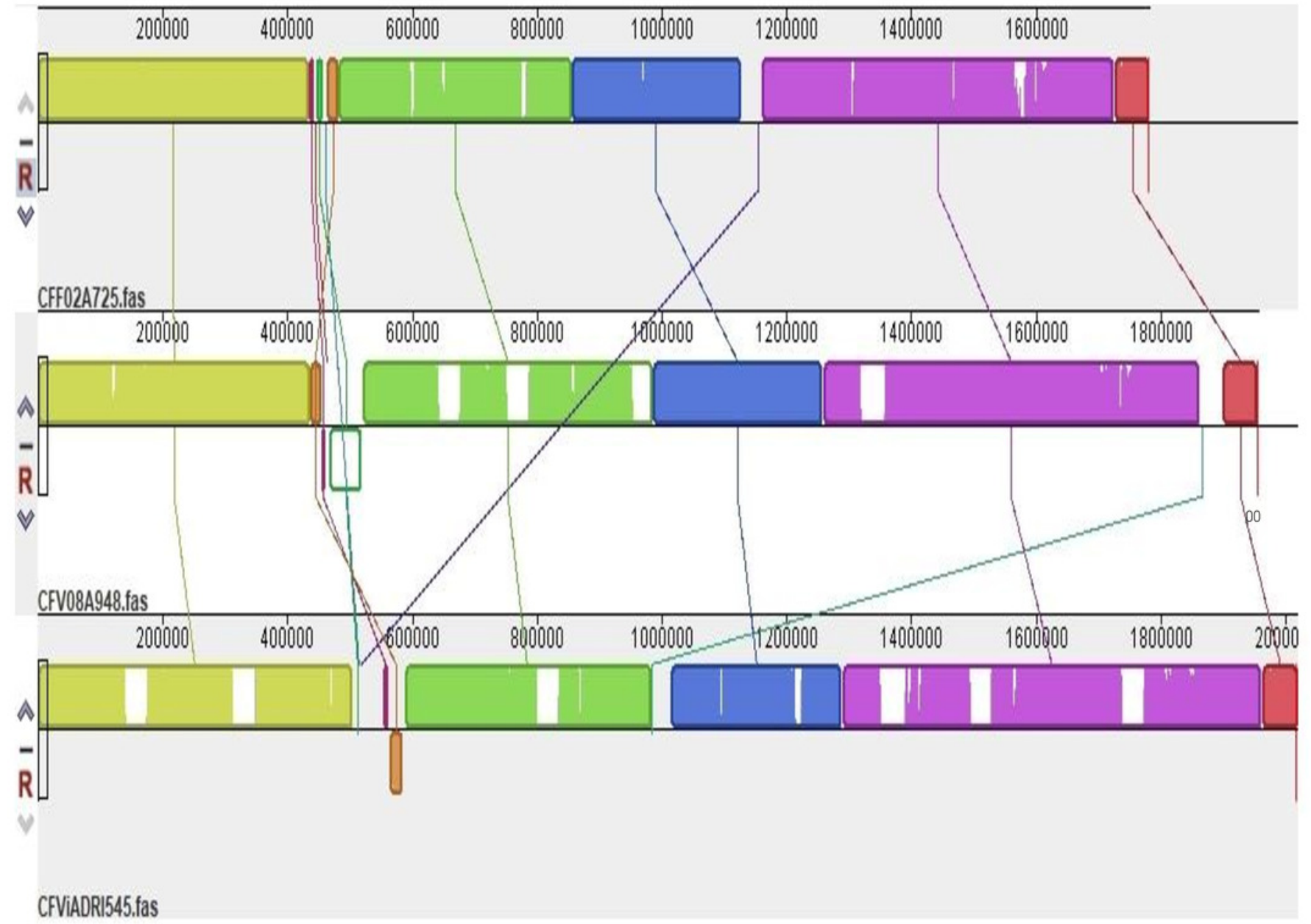


Figure 2

\section{Alignment of six CFF genomes}

Assembled genomes were aligned using the progressive Mauve option of Mauve software v2.3.1.

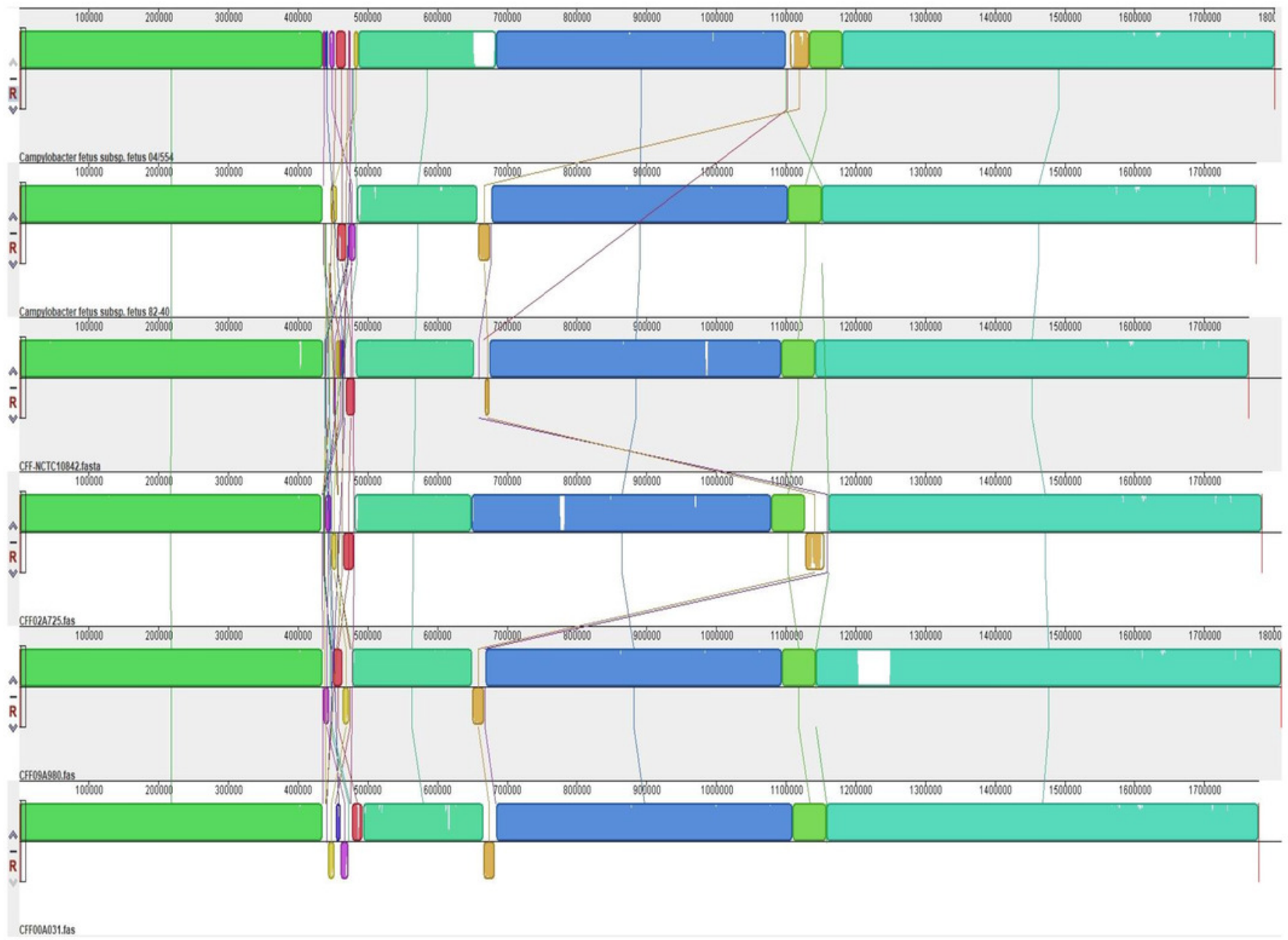


Figure 3

\section{Alignment of five CFV genomes}

Assembled genomes were aligned using the progressive Mauve option of Mauve software v2.3.1.

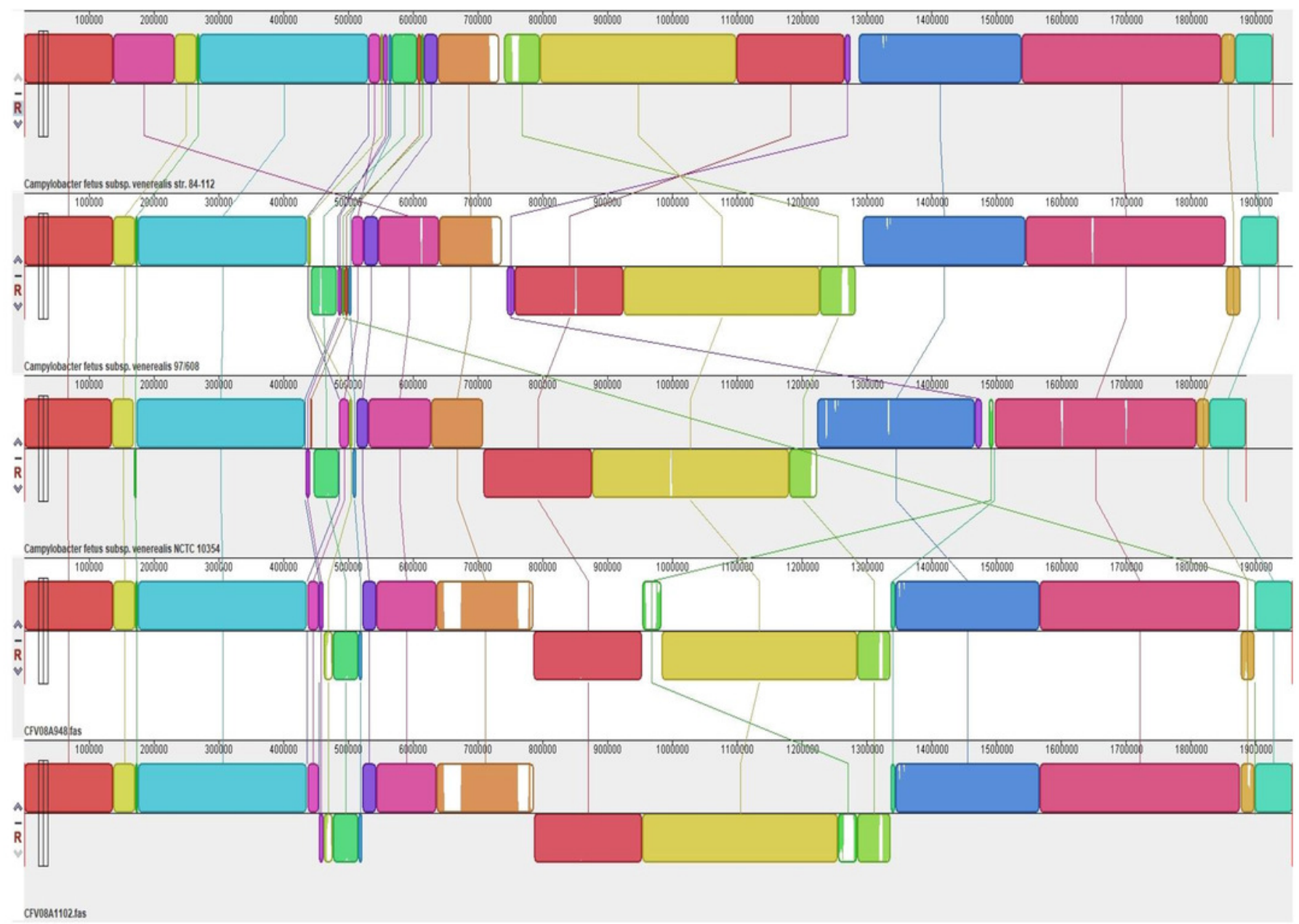


Figure 4

\section{Alignment of three CFVi genomes}

Assembled genomes were aligned using the progressive Mauve option of Mauve software v2.3.1.

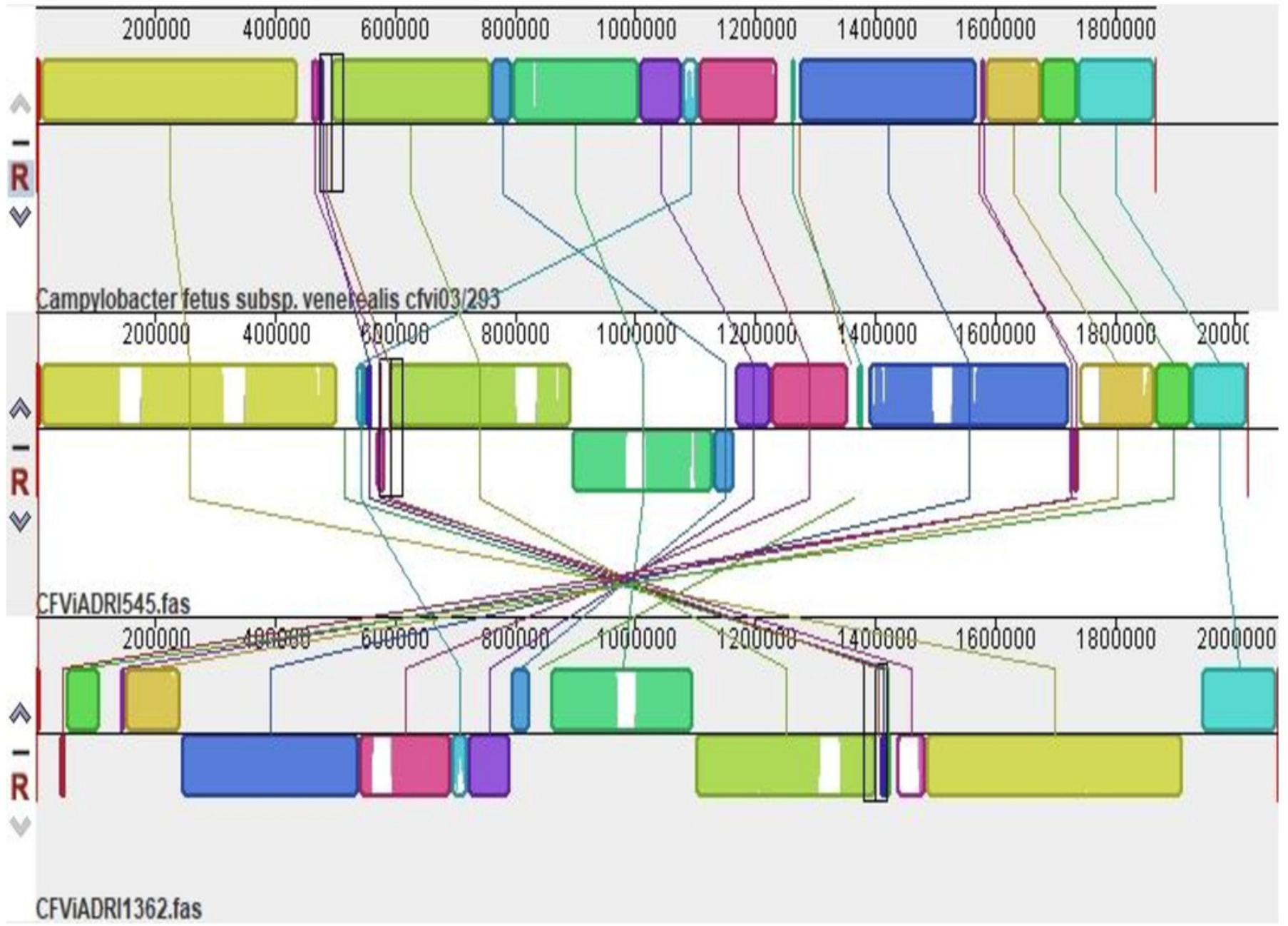




\section{Figure 5}

Schematic of the hypervariable region for $14 \mathrm{CF}$ isolates

Arrows, which are not drawn to scale, indicate the gene orientation. Squares indicate either hypothetical or pseudo genes. Grey arrows at the termini indicate genes common to all isolates, i.e. an upstream diheme cytochrome $\mathrm{c}$ and a downstream glycosyl transferase (gtf). Other colour codes represent genes thus: black, sapA; brown, sapB; emerald green, an 11 gene cassette comprising ssrA (which encodes a tmRNA), a histidine phosphotransferase, panB, a sulfur transferase, ruvB, amaA, mlaE, mlaF, mlaD, mlaB and ybeY (an endoribonuclease); purple, a four gene cassette comprising tolC, prsE, prsD and a peptidase; yellow, a five gene set comprising gtf, $m t f A$, a hypothetical product and $A B C$ transporter and permease genes; pink, yafQ; orange, AAA family ATPase; blue, hipA; light green, surface protein; white (unlabelled), transporters of variable sequence; turquoise, lexA, a repressor at the boundary of most prophages; additional novel genes are indicated by white arrows and labelled individually. Red circles identify IS605 elements. The numbers below each schematic indicate the start and stop locations of the terminal gene ORFs. Note that the CFVi-ADRI1362 schematic is shown in reverse orientation relative to the others. Samples CFV-08A948 and CFV-08A1102 were identical except for the inversion of the purple cassette in the latter. 

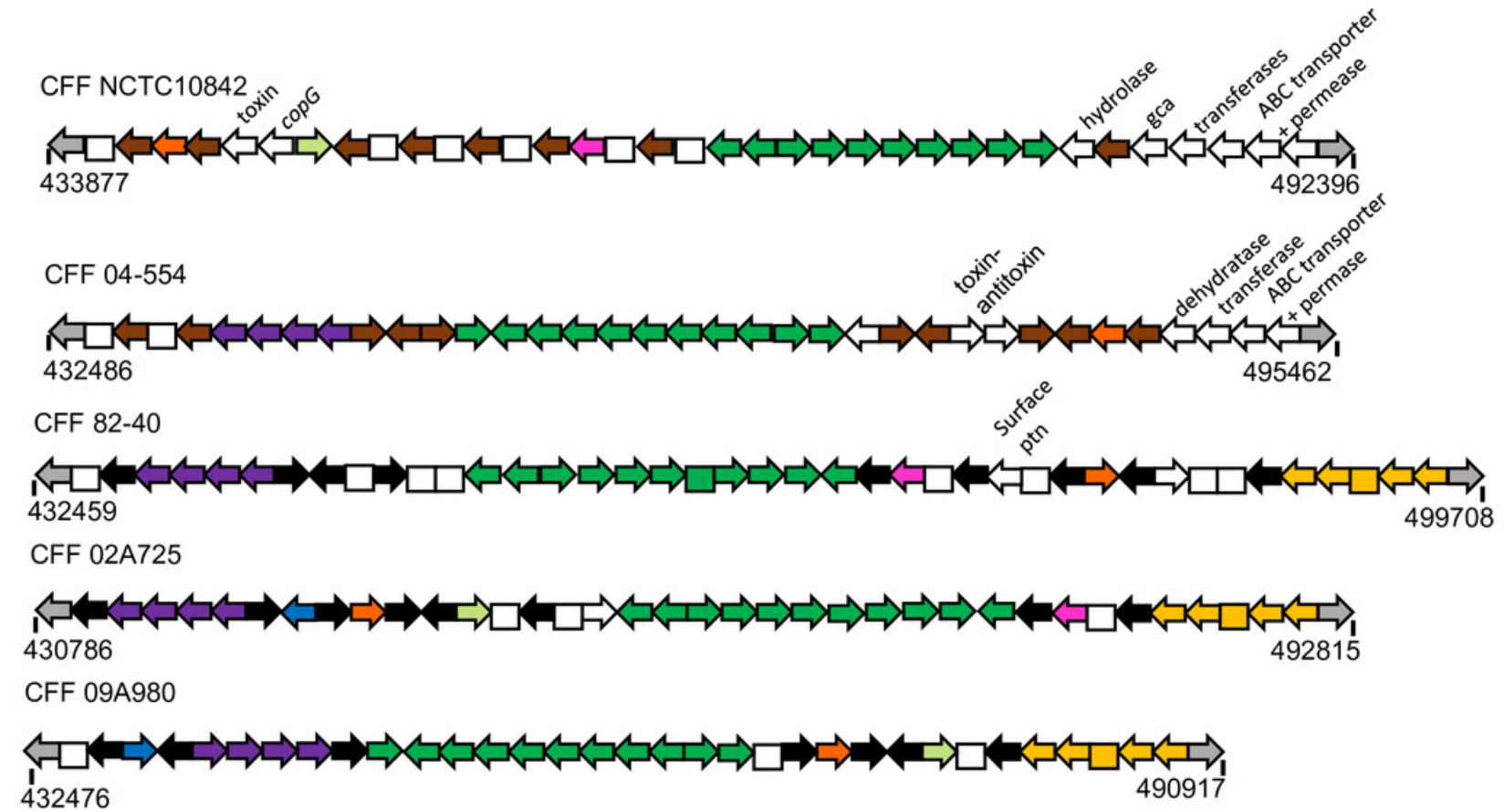

CFF 00A031 ${ }_{432475}$

CFV NCTC10354

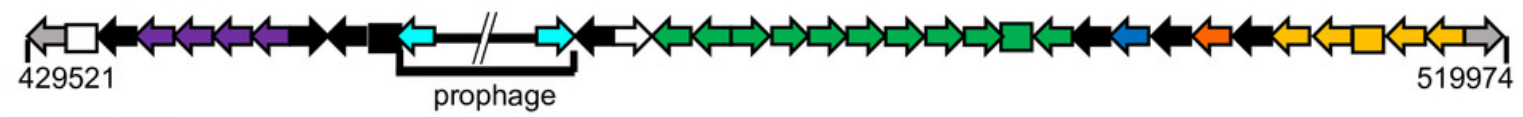

CFV 84-112
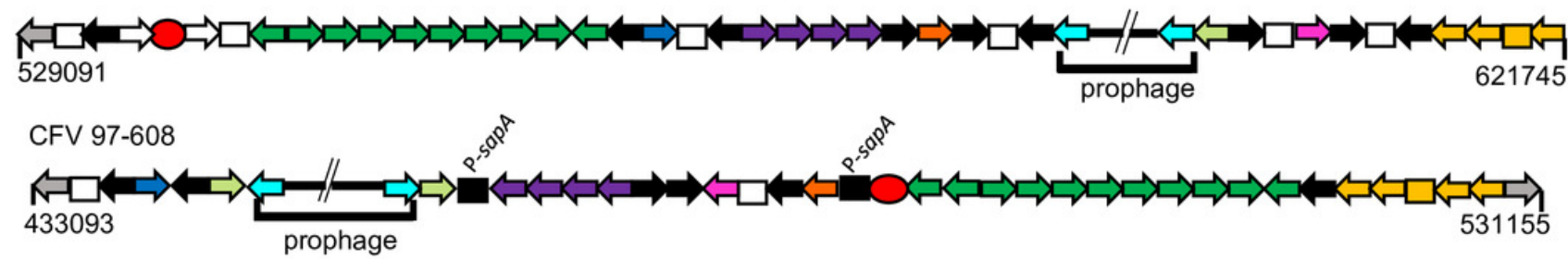

CFV 08A948/08A1102

${ }_{433096}$

CFVi 03-293

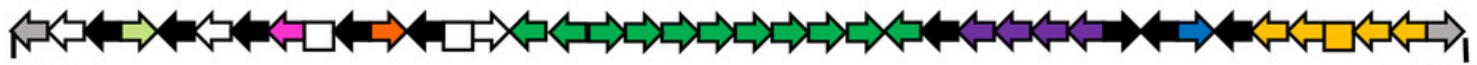
433184

CFVi ADRI545

501713

CFVi ADRI1362

1487351 


\section{Figure 6}

Schematics of representatives of the five types of T4SS genomic island found in CFs

Arrows indicate the gene orientation; hypothetical genes are represented by boxes, white for singles and black for multiple. Blue arrows indicate Type IV SS members though in some cases there was significant variation in the primary sequence of some alleles. Other genes shown in white are frequently plasmid-associated. Red circles indicate IS605 elements. The range of each $\mathrm{Gl}$ is indicated below each schematic.

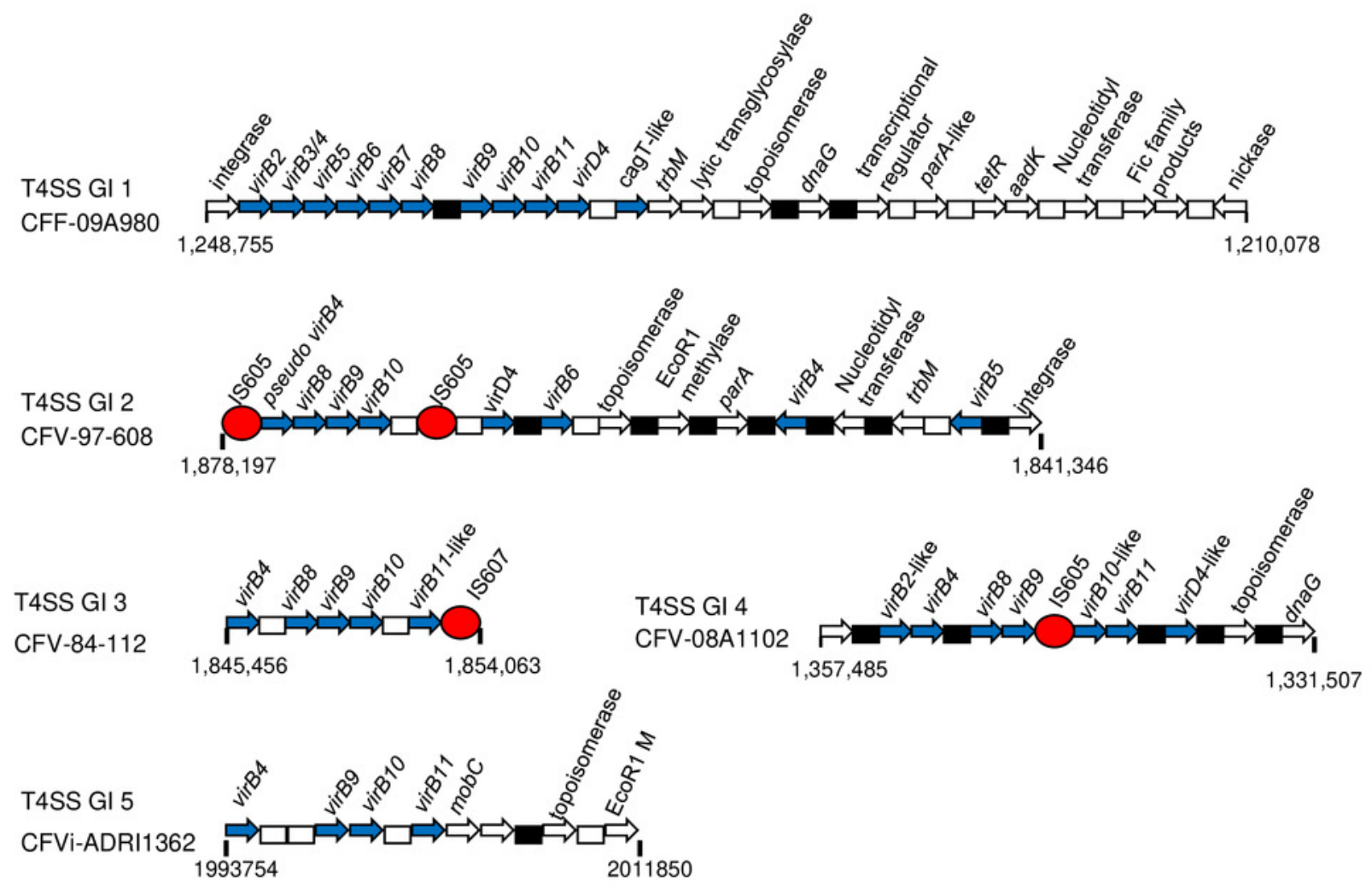




\section{Table $\mathbf{1}$ (on next page)}

Characteristics of the fourteen $C$. fetus isolates compared in this study 
Tablle 1. Characteristics of the fourteen C. fetus isolates compared in this study

\begin{tabular}{|c|c|c|c|c|c|}
\hline $\begin{array}{l}\text { Sample } \\
\text { designation }\end{array}$ & Country of origin & $\begin{array}{l}\text { Year of } \\
\text { isolation }\end{array}$ & Source details & Serotype & Phenotypic identification \\
\hline \multicolumn{6}{|c|}{ Samples analysed in this study } \\
\hline CFF-02A725 & Canada - Alberta & 2002 & Bovine, Preputial wash & A & C. fetus fetus \\
\hline CFF-09A980 & Canada - Quebec & 2009 & Bovine, Preputial wash & A & C. fetus fetus \\
\hline CFF-00A031 & Canada - British Columbia & 2000 & Bovine, Preputial wash & A & C. fetus fetus \\
\hline CFV-08A948 & Canada-Alberta (Premise 1) & 2008 & Bovine, Preputial wash & A & C. fetus venerealis \\
\hline CFV-08A1102 & Canada - Alberta (Premise 2) & 2008 & Bovine, Preputial wash & A & C. fetus venerealis \\
\hline CFVi-ADRI545 & Northern Australia & 1984 & Bovine, Reproductive tract & $\mathrm{A}$ & C. fetus venerealis biovar intermedius \\
\hline CFVi-ADRI1362 & Argentina & 1989 & Bovine, Vaginal mucus & $\mathrm{A}$ & C. fetus venerealis biovar intermedius \\
\hline \multicolumn{6}{|c|}{ Samples analysed in previous studies } \\
\hline CFF-82-40 & USA & 1982 & Human & A & C.fetus fetus \\
\hline CFF-04-554 & Argentina & 2004 & Bovine fetus & $\mathrm{B}$ & C. fetus fetus \\
\hline CFF-NCTC10842 & France & unknown & Ovine, aborted fetus & $\mathrm{B}$ & C. fetus fetus \\
\hline CFV-84-112 & USA & 1984 & Bovine & A & C. fetus venerealis \\
\hline CFV-97-608 & Argentina & 1987 & Bovine & A & C. fetus venerealis \\
\hline CFV-NCTC10354 & United Kingdom & 1952 & Bovine, heifer vaginal mucus & A & C. fetus venerealis \\
\hline CFVi-03-293 & Argentina & 2003 & Bovine, aborted fetus lung & $\mathrm{A}$ & C. fetus venerealis biovar intermedius \\
\hline
\end{tabular}




\section{Table 2 (on next page)}

Characteristics of the chromosomal genomes of the fourteen $C$. fetus isolates compared in this study

${ }^{1}$ including pseudogenes ${ }^{2}$ Data recovered from Van der Graaf, 2014 In this and all subsequent tables solid horizontal lines separate CFF, CFV and CFVi isolates while a dotted horizontal line separates serotype A and B CFFs 
2 Table 2. Characteristics of the chromosomal genomes of the fourteen C. fetus isolates compared in this study

\begin{tabular}{lllllll}
\hline Isolate designation & $\begin{array}{l}\text { Genome length } \\
\text { (bp) }\end{array}$ & $\begin{array}{l}\text { \% GC } \\
\text { content }\end{array}$ & $\begin{array}{l}\text { No. of } \\
\text { Genes }^{1}\end{array}$ & $\begin{array}{l}\text { No. of } \\
\text { rRNA } \\
\text { operons }\end{array}$ & $\begin{array}{l}\text { No. of } \\
\text { tRNA loci }\end{array}$ & $\begin{array}{l}\text { NCBI Assembly } \\
\text { Accession \# }\end{array}$ \\
\hline CFF-82-40 & $1,773,615^{2}$ & 33.3 & 1769 & 3 & 43 & CP000487 \\
CFF-02A725 & $1,782,312$ & 33.3 & 1872 & 3 & 44 & CP059974 \\
CFF-09A980 & $1,810,730$ & 33.3 & 1838 & 3 & 44 & CP059445 \\
CFF-00A031 & $1,777,789$ & 33.3 & 1797 & 3 & 44 & CP059443 \\
\hline CFF-04-554 & $1,800,764^{2}$ & 33.2 & 1752 & 3 & 43 & CP008808 \\
CFF-NCTC10842 & $1,763,253$ & 33.3 & 1796 & 3 & 44 & LS483431 \\
\hline CFV-84-112 & $1,926,886^{2}$ & 33.3 & 1992 & 3 & 43 & HG004426 \\
CFV-97-608 & $1,935,028^{2}$ & 33.3 & 1939 & 3 & 43 & CP008810 \\
CFV-NCTC10354 & $1,885,704$ & 33.2 & 1914 & 3 & 44 & CP043435 \\
CFV-08A948 & $1,956,863$ & 33.3 & 2046 & 3 & 44 & CP059441 \\
CFV-08A1102 & $1,956,865$ & 33.3 & 2048 & 3 & 44 & CP059439 \\
\hline CFVi-03-293 & $1,866,009^{2}$ & 33.3 & 1821 & 3 & 43 & CP006999 \\
CFVi-ADRI545 & $2,019,872$ & 33.4 & 2156 & 3 & 44 & CP059437 \\
CFVi-ADRI1362 & $2,071,525$ & 33.6 & 2237 & 3 & 44 & CP059432 \\
\hline
\end{tabular}

$3 \quad$ including pseudogenes

$4 \quad 2$ Data recovered from Van der Graaf, 2014

5 In this and all subsequent tables solid horizontal lines separate CFF, CFV and CFVi isolates while a dotted horizontal line separates 6 serotype A and B CFFs 


\section{Table 3(on next page)}

Summary of transposons and prophages identified in $C$. fetus genomes

** indicates truncated product 
Table 3. Summary of transposons and prophages identified in C. fetus genomes

\begin{tabular}{|c|c|c|c|c|c|c|}
\hline & \multicolumn{2}{|r|}{ IS605 element } & \multicolumn{2}{|r|}{ IS607 element } & \multicolumn{2}{|r|}{ Prophage sequence } \\
\hline & $\begin{array}{l}\text { copy } \\
\text { number }\end{array}$ & location & $\begin{array}{l}\text { copy } \\
\text { number }\end{array}$ & location & $\begin{array}{c}\text { copy } \\
\text { number }\end{array}$ & $\begin{array}{c}\text { location of phage portal } \\
\text { protein }\end{array}$ \\
\hline CFF-82-40 & 0 & & 0 & & 1 & $660,498-661,559$ \\
\hline CFF-02A725 & 0 & & 0 & & 1 & $\begin{array}{l}1,140,631-1,140,831 * * \\
1,141,256-1,141,690 * *\end{array}$ \\
\hline CFF-09A980 & 0 & & 0 & & 1 & $651,841-652,902$ \\
\hline CFF-00A031 & 0 & & 0 & & 1 & $667,640-668,701$ \\
\hline CFF-04-554 & 0 & & 0 & & 1 & $1,119,046-1,120,107$ \\
\hline CFF-NCTC10842 & 0 & & 0 & & 1 & $670,040-671,101$ \\
\hline CFV-84-112 & 2 & $\begin{array}{c}533,924-535,307 \\
1,868,521-1,870,054\end{array}$ & 0 & & 3 & $\begin{array}{l}585,408-586,472 ; \\
739,112-740,185 ; \\
1,277,904-1,278,977\end{array}$ \\
\hline CFV-97-608 & 3 & $\begin{array}{c}507,749-509,438 ; \\
850,118-851,810 ; \\
1,876,554-1,878,246\end{array}$ & 6 & $\begin{array}{c}120,302-122,369 ; \\
456,444-458,511 ; \\
611,586-613,653 ; \\
827,189-829,256 ; \\
1,647,029-1,649,096 ; \\
1,869,088-1,871,155\end{array}$ & 3 & $\begin{array}{l}464,286-465,362 ; \\
743,287-744,360 ; \\
1,283,953-1,285,026\end{array}$ \\
\hline CFV-NCTC10354 & 3 & $\begin{array}{l}1,236,767-1,238,459 \\
1,332,720-1,334,412 \\
1,699,299-1,700,991\end{array}$ & 3 & $\begin{array}{l}779,640-781,707 \\
996,739-998,806 \\
1,599,993-1,602,060\end{array}$ & 1 & $466,844-467,920$ \\
\hline CFV-08A948 & 5 & $\begin{array}{l}439,037-440,570 \\
1,343,995-1,345,528 \\
1,734,964-1,736,497 \\
1,874,884-1,876,417 \\
1,898,499-1,900,032\end{array}$ & 3 & $\begin{array}{l}\text { 120,406-122,323; } \\
857,035-858,952 ; \\
1,890,970-1,892,887\end{array}$ & 4 & $\begin{array}{l}496,550-497,626 ; \\
654,579-655,652 ; \\
773,210-774,283 \\
965,366-966,439\end{array}$ \\
\hline
\end{tabular}




\begin{tabular}{|c|c|c|c|c|c|c|}
\hline CFV-@8A1102 & 5 & $\begin{array}{l}439,037-440,570 \\
1,343,999-1,345,532 \\
1,734,965-1,736,498 \\
1,874,886-1,876,419 \\
1,898,501-1,900,034\end{array}$ & 3 & $\begin{array}{l}\text { 120,406-122,323; } \\
857,221-859,138 ; \\
1,882,031-1,883,948\end{array}$ & 4 & $\begin{array}{l}496,551-497,627 ; \\
654,580-654,792^{* *}+ \\
655,217-655,654^{* *} \\
773,214-774,287 ; \\
1,272,652-1,273,725\end{array}$ \\
\hline CFVi-03-293 & 3 & $\begin{array}{l}\text { 1,006,929-1,008,846; } \\
1,094,859-1,096,392 \\
1,249,187-1,250,720\end{array}$ & 3 & $\begin{array}{l}420,725-422,642 ; \\
758,891-760,808 ; \\
1,083,526-1,085,433 *\end{array}$ & 1 & $1,088,131-1,089,192$ \\
\hline CFVi-ADRI545 & 4 & $\begin{array}{l}\text { 580,856-582,389; } \\
868,430-869,963 ; \\
1,094,766-1,096,299 \\
1,358,484-1,360,018\end{array}$ & 4 & $\begin{array}{l}\text { 1,395,744-1,397,661; } \\
1,411,977-1,413,894 \\
1,564,281-1,566,198 \\
1,738,304-1,740,221\end{array}$ & 7 & $\begin{array}{l}\text { 161,401-162,462; } \\
325,552-326,613 ; \\
536,549-537,625 ; \\
821,342-822,403 ; \\
996,953-998,014 ; \\
1,513,357-1,514,418 ; \\
1,752,790-1,753,851\end{array}$ \\
\hline CFVi-ADRI1362 & 0 & & 5 & $\begin{array}{l}\text { 47,649-49,566; } \\
707,540-709,457 ; \\
790,038-791,955 ; \\
\text { 1,098,560-1,100,477; } \\
1,498,399-1,500,316\end{array}$ & 9 & $\begin{array}{l}\text { 20,800-21,861; } \\
\text { 128,294-129,355; } \\
\text { 573,651-574,712; } \\
\text { 711,744-712,805; } \\
\text { 838,573-839,634; } \\
\text { 981,695-982,768; } \\
\text { 1,320,740-1,321,801; } \\
\text { 1,452,009-1,453,070; } \\
1,930,918-1,931,979\end{array}$ \\
\hline
\end{tabular}

** truncated product 


\section{Table 4(on next page)}

Summary of the CRISPR-cas loci present in 14 C. fetus isolates

* sequence extended at $5^{\prime}$ end;

** truncated sequence;

(P) pseudogene 
Table 4. Summary of the CRISPR-cas loci present in 14 C. fetus isolates

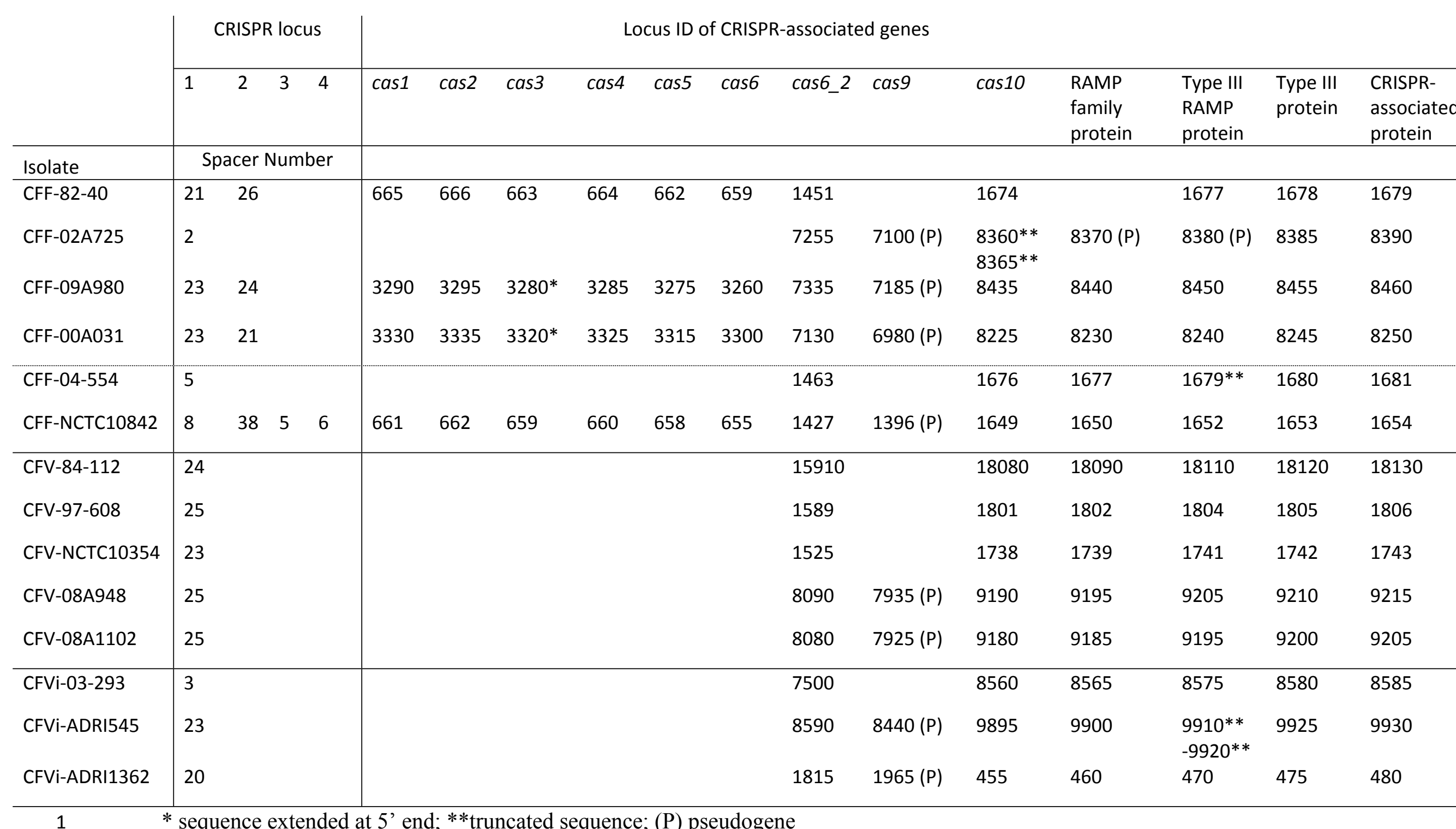




\section{Table 5 (on next page)}

Type IV Secretion System Genomic islands in 14 C. fetus isolates

* integrase to lytic transglycosylase only

** Integrase to dnaG only with highly modified product sequences 
1 Table 5. Type IV Secretion System Genomic islands in 14 C. fetus isolates

\begin{tabular}{|c|c|c|c|c|c|c|c|c|c|c|}
\hline & \multicolumn{2}{|c|}{ T4SS GI 1} & \multicolumn{2}{|c|}{ T4SS GI 2} & \multicolumn{2}{|c|}{ T4SS GI 3} & \multicolumn{2}{|c|}{ T4SS GI 4} & \multicolumn{2}{|c|}{ T4SS GI 5} \\
\hline & $\begin{array}{c}\text { copy } \\
\text { number }\end{array}$ & location & $\begin{array}{c}\text { copy } \\
\text { number }\end{array}$ & location & $\begin{array}{c}\text { copy } \\
\text { number }\end{array}$ & location & $\begin{array}{c}\text { copy } \\
\text { number }\end{array}$ & location & $\begin{array}{c}\text { copy } \\
\text { number }\end{array}$ & location \\
\hline CFF-82-40 & 0 & & & & & & & & & \\
\hline CFF-02A725 & 0 & & & & & & & & & \\
\hline CFF-09A980 & 1 & $\begin{array}{c}1,203,385- \\
1,252,250\end{array}$ & & & & & & & & \\
\hline CFF-00A031 & 0 & & & & & & & & & \\
\hline CFF-04-554 & 0 & & & & & & & & & \\
\hline CFF-NCTC10842 & 0 & & & & & & & & & \\
\hline CFV-84-112 & 1 & $\begin{array}{l}1,300,266- \\
1,330,033\end{array}$ & & & 1 & $\begin{array}{l}1,845,456- \\
1,854,063\end{array}$ & & & & \\
\hline CFV-97-608 & 1 & $\begin{array}{l}1,306,338- \\
1,336,107\end{array}$ & 1 & $\begin{array}{l}1,841,346- \\
1,878,197\end{array}$ & & & & & & \\
\hline CFV-NCTC10354 & 1 & $\begin{array}{c}1,224,281- \\
1,255,740\end{array}$ & & & & & & & & \\
\hline CFV-08A948 & 0 & & & & & & 1 & $\begin{array}{l}1,331,503- \\
1,357,485\end{array}$ & & \\
\hline CFV-08A1102 & 0 & & & & & & 1 & $\begin{array}{l}1,331,507- \\
1,357,489\end{array}$ & & \\
\hline CFVi-03-293 & 1 & $\begin{array}{l}1,250,766- \\
1,272,991^{*}\end{array}$ & & & & & & & 1 & $\begin{array}{l}1,788,240- \\
1,806,336\end{array}$ \\
\hline CFVi-ADRI545 & 1 & $\begin{array}{r}1,358,484- \\
1,389,267^{* *}\end{array}$ & & & & & & & & \\
\hline CFVi-ADRI1362 & 0 & & & & & & & & 1 & $\begin{array}{l}1,993,754- \\
2,011,850\end{array}$ \\
\hline
\end{tabular}

$2 *$ integrase to lytic transglycosylase only

$3 * *$ Integrase to $d n a G$ only with highly modified product sequences 
Table 6(on next page)

Listing of the plasmids found in 11 of $14 \mathrm{C}$. fetus isolates

Annotations of all plasmids are provided in Supplementary Table S4. 
1 Table 6. Listing of the plasmids found in 11 of 14 C. fetus isolates

\begin{tabular}{llll}
\hline Isolate & $\begin{array}{l}\text { Plasmid, size } \\
(\mathrm{bp})\end{array}$ & $\begin{array}{l}\text { Number } \\
\text { of genes }\end{array}$ & $\begin{array}{l}\text { NCBI Accession } \\
\text { number }\end{array}$ \\
\hline CFF-82-40 & & & \\
CFF-02A725 & P1, 52,345 & 66 & CP059446 \\
CFF-09A980 & P1, 26,793 & 36 & CP059444 \\
CFF-00A031 & P1, 25,862 & 32 & CP008809 \\
\hline CFF-04-554 & & & \\
CFF-NCTC10842 & P1, 61,142 & 73 & HG004427 \\
\hline CFV-84-112 & P1, 38,272 & 43 & CP008811. \\
CFV-97-608 & P2, 27,124 & 35 & CP008812 \\
& P1, 27,915 & 36 & CP043436 \\
CFV-NCTC10354 & P1, 38,770 & 50 & CP059442 \\
CFV-08A948 & P1, 37,205 & 49 & CP059440 \\
CFV-08A1102 & P1, 91,400 & 124 & CP007000 \\
\hline CFVi-03-293 & P2, 35,326 & 38 & CP007001 \\
& P3, 3,993 & 6 & CP007002 \\
& P1, 48,693 & 68 & CP059438 \\
CFVi-ADRI545 & P1, 40,588 & 58 & CP059433 \\
CFVi-ADRI1362 & P2, 36,566 & 36 & CP059434 \\
& P3, 35,640 & 38 & CP059435 \\
& P4, 3,993 & 5 & CP059436 \\
\hline
\end{tabular}

2

3 Annotations of all plasmids are provided in Supplementary Table S4. 